\title{
DEPARTMENT OF THE INTERIDR
}

U.S. GEOLOGICAL SURVEY

\begin{abstract}
Sample localities, radiometric ages, descriptions, and major- and trace-element abundances of Late Jurassic mafic plutonic rocks, eastern Sierra Nevada, California
\end{abstract}

by

Thomas P. Frost

MS 938

345 Middlefield Road

Menlo Park, California 94025

Open-File Report 87-484

This report is preliminary and has not been reviewed for conformity with U.S. Geological Survey editorial standards (and stratigraphic nomenclature). Trade names used are for identification only and do not constitute an endorsement by the U.S. Geological Survey. 
Introduction

This report is a compilation of sample localities, radiometric ages, abbreviated sample descriptions, and major- and trace-element determinations of samples from selected mafic plutons from the eastern Sierra Nevada, California, between $36^{\circ} 45^{\prime} \mathrm{N}$ and $37^{\circ} 35^{\prime} \mathrm{N}$. Figure 1 is a map of the region under consideration, Figures 2 through 20 are major and trace element Harker plots for the oxides and trace elements vs. silica. Table 1 describes the localities and rock types present at each locality, Table 2 presents sample locality latitude and longitudes for the samples on which major- and trace-element determinations were made. Table 3 presents the major element data. Table 4 presents minor- and trace-element data. Table 5 presents the radiometric age data for mafic rocks of the eastern Sierra Nevada. Fifteen minute USGS quadrangle maps covered are: Casa Diablo Mountain, Mt. Abbot, Mt. Tom, Bishop, Mt. Goddard, Big Pine, Marion Peak, and Mt. Pinchot. Geochemical data for Late Cretaceous mafic intrusions associated with the Lamarck Granodiorite (D and $H$ on Fig. 1) are presented in Frost (1987) and Frost and Mahood (1987).

Geological overview

Numerous calc-alkalic gabbroic through quartz dioritic mafic intrusive complexes are present in the eastern Sierra Nevada of California. The bodies range from discrete intrusive bodies with outcrop areas as large as $25 \mathrm{~km}^{2}$ to remobilized discontinuous screens and septa between younger felsic plutons. Many of the mafic intrusive complexes may have been more areally extensive prior to disruption caused by emplacement of younger granitoids. The intrusions are compositionally and lithologically heterogeneous and range from distinctively layered olivine-clinopyroxene-hornblende gabbros through hornblende 
gabbro to biotite-hornblende diorite and quartz diorite. Anorthosite and peridotite are locally present.

Mafic intrusions believed to be as young as Late Cretaceous are relatively abundant in the eastern Sierra Nevada batholith (Frost and Mahood, 1987, Frost and Mattinson, unpub data); mafic intrusions considered in detail in this report are present exclusively in and among granitoids of Jurassic and Triassic age in the eastern part of the Sierra Nevada batholith (Fig. 1).

\section{Sampling Methods}

All coarse grained samples collected in the field weighed at least $5 \mathrm{~kg}$; samples taken from areas accessible by automobile weighed at least $20 \mathrm{~kg}$. Samples were coarsely crushed to thumb-joint size with a steel sledge hammer with the sample sandwiched between at least 5 sheets of blank computer paper. After coarse crushing, the samples were inspected visually and any fragments of computer paper adhering to them was removed. A small tungsten carbide jaw crusher was then used to reduce the samples to $1-3 \mathrm{~mm}$. The interior and exterior of the crusher was scrubbed with a toothbrush and thoroughly blasted with filtered compressed air between each sample. The first several handfuls of each sample passed through the crusher were discarded. After jaw crushing, each sample was repeatedly split in a baffle-splitter to yield a sample of about $70 \mathrm{~g}$.

A tungsten carbide shatterbox was used to pulverize the samples to approximately -200 mesh. Between each sample, the shatterbox was scrubbed with a toothbrush, washed in tap water, and dried with compressed air. Final cleaning was performed by crushing a split of the next sample for one minute. The shatterbox was then emptied, brushed with a clean toothbrush, rinsed in deionized water, and dried before the split to be analysed was loaded for crushing. Crushing times varied between 3 and 5 minutes, with biotite-rich 
samples requiring the longer times. Quartz subjected to the same procedure showed measurable contamination in $\mathrm{Co}_{0}$ and $\mathrm{W}$; contamination by other elements was below detection limits for energy-dispersive $x$-ray fluorescence (XRF) spectrometry.

\section{$X-R a y$ Fluorescence Spectroscopic Analys is}

Major-element determinations were made by standard wavelength-dispersive XRF spectroscopic techniques. Reported abundances were made by averaging two determinations on replicate fused glass discs made with lithium tetraborate flux. A sample-flux ratio of 1:10 was utilized. The complete fusion technique is described in Taggart et al (1987). Determinations were made on a Diano 8600 wavelength-dispersive XRF spectrometer. Calibration curves for the major oxides were made by standard methods described by Taggart et al (1987).

Vanadium abundances reported were made by standard wavelength-dispersive XRF spectroscopic techniques using pressed powder pellets on a Diano 8600 spectrometer. Reported values are averages of at least three determinations per sample. An 85:15 ratio of sample powder to spec-pure methyl cellulose binder was used in the pellets. Sample preparation methods and calibration techniques are described in Fabbi (1970), King et al (1977), and Taggart et al (1987) .

Minor- and trace-element abundances reported, other than vanadium, are averages of triplicate determinations on replicate powders made on a Kevex 0700/7000 energy-dispersive $x$-ray spectrometer; matrix corrections were based on Compton-scatter radiation (Johnson, 1984).

\section{Acknowledgments}

Reviewed by B.W. King and D.V. Vivit. 
Bateman PC (1965) Geology and tungsten mineralization of the Bishop district, California. U.S. Geol. Surv. Prof. Pap. 470, 208 p.

Bateman PC, Moore JG (1965) Geologic map of the Mt. Goddard quadrangle, California U.S. Geological Survey Geologic Quadrangle Map GQ-429.

Chen JH, Moore JG (1979) Late Jurassic Independence dike swarm in eastern California. Geology, v. 7, p. 129-133.

Chen JH, Moore JG (1982) Uranium-lead isotopic ages from the Sierra Nevada batholith, California. Journal of Geophysical Research, v.87B, p. $4761-4784$.

Dodge FCW, Moore JG (1968) 0ccurrence and composition of biotites from the Cartridge Pass pluton of the Sierra Nevada batholith, California. U.S. Geological Survey Professional Paper 600-B, B6-B10.

Evernden JF, Kistler, RW (1970) Chronology of emplacement of Mesozoic batholithic complexes of California and western Nevada. U.S. Geological Survey Professional Paper 623, $42 \mathrm{p}$.

Fabbi BF (1970) A die for pelletizing samples for $x$-ray fluorescence analysis. U.S. Geological Survey Prof. Pap. 700-B, B187-B189.

Frost TP (1987) Sample localities, descriptions, major- and trace-element abundances from the Lamarck Granodiorite and associated mafic rocks, Sierra Nevada, California. U. S. Geological Survey Open-File Report $87-193,38 \mathrm{p}$.

Frost TP, Mahood GA (1987) Field, chemical, and physical constraints on maficfelsic magma interaction in the Lamarck Granodiorite, Sierra Nevada, California. Geological Society of America Bulletin, v. 99, p. 272-291. Johnson RG (1984) Trace element analysis of silicates by means of energy-dispersive $X-r$ ay spectrometry. X-ray Spectroscopy, v.13, p. 64-68.

King BS, Espos LF, Fabbi BP (1977) X-ray fluorescence minor- and trace-element analysis of silicate rocks in the presence of large interelement effects. Advances in X-ray Analysis, v. 21, p. 75-88.

Lockwood JP, Lydon, PA (1975) Geologic map of the Mount Abbot quadrangle, central Sierra Nevada, California. U.S. Geological Survey Map GQ-1155.

Moore JG (1963) Geology of the Mt. Pinchot quadrangle, southern Sierra Nevada, California. U.S. Geol. Surv. Bulletin 1130, 152 p.

Moore JN, Foster CT, jr (1980) Lower Paleozoic metasedimentary rocks in the east central Sierra Nevada, Cal ifornia: correlations with Great Basin formations. Geol. Soc. Amer. Bull., v. 91, 37-43.

Rinehart CD, Ross DC (1957) Geology of the Casa Diablo Mountain quadrangle, Mono County, California. U.S. Geological Survey Map GQ-99.

Rinehart CD, Ross DC (1964) Geology and mineral deposits of the Mount Morrison quadrangle, Sierra Nevada, California. U.S. Geological Survey Professional Paper 385, 106 p.

Sawka WN (1985) The geochemistry of differentiation processess in granite magma chambers. unpub Ph.D. dissertation, Australian National University.

Stern TW, Bateman PC, Morgan BA, Newell BA, Peck DL (1981) Isotopic U-Pb ages of zircon from granitoids of the central Sierra Nevada, California. U.S. Geol. Surv. Prof. Pap. 1185, 17 p.

Taggart JE jr, Lindsay JR, Scott BA, Vivit DA, Bartel AJ, Stewart KC (1987) Analysis of geological materials by wavelength dispersive $x$-ray

fluorecense spectroscopy. Chapter 5 in U.S. Geol. Surv. Bullet in 1770, in press.

Tobisch OT, Saleeby JB, Fiske RS (1986) Structural history of continental volcanic arc rocks, eastern Sierra Nevada, California: a case for extensional tectonics. Tectonics, v. 5, p. 65-94. 


\section{FIGURE CAPTIONS}

Fig. 1. Location and regional geologic map. Sources: Rinehart and Ross, 1957, 1964; Moore, 1963; Bateman, 1965; Bateman and Moore, 1965; Moore and Foster, 1980; Stern et al, 1981; Chen and Moore, 1979, 1982; Frost and Mahood, 1987; Frost and Mattinson, unpub. data; Tobisch et al, 1986)

Fig. 2. Harker diagram, Ti02, in weight percent. All data recalculated anhydrous. Locality coding: A - Armstrong Canyon; C - Casa Diablo-Rock Creek; K - Keough Hot Springs; M - McMurry Meadows, felsic layers; Z - McMurry Meadows, mafic layers; S - Thibaut-Black Canyon; T - Tungsten Hills; X Mt. Tom.

Fig. 3. Harker diagram, A1203. All data as in Fig. 2.

Fig. 4. Harker diagram, $\mathrm{Fe}^{*}(\mathrm{All}$ iron recalculated as $\mathrm{FeO})$. All data as in Fig. 2.

Fig. 5. Harker diagram, Mg0. All data as in Fig. 2.

Fig. 6. Harker diagram, MnO. All data as in Fig. 2.

Fig. 7. Harker diagram, CaO. All data as in Fig. 2.

Fig. 8. Harker diagram, Na20. All data as in Fig. 2.

Fig. 9. Harker diagram, K20. All data as in Fig. 2.

Fig. 10. Harker diagram, P205. All data as in Fig. 2.

Fig. 11. Harker diagram, Ba (in ppm). All other data as in Fig. 2.

Fig. 12. Harker diagram, $\mathrm{Cr}$ (in ppm). Samples shown as zero did not have $\mathrm{Cr}$ determined. All other data as in Fig. 2 .

Fig. 13. Harker diagram, ND (in ppm). All other data as in Fig. 2.

Fig. 14. Harker diagram, Ni (in ppm). All other data as in Fig. 2.

Fig. 15. Harker diagram, Rb (in ppm). All other data as in Fig. 2.

Fig. 16. Harker diagram, Sr (in ppm). All other data as in Fig. 2.

Fig. 17. Harker diagram, $V$ (in ppm). All other data as in Fig. 2.

Fig. 18. Harker diagram, $Y$ (in ppm). All other data as in Fig. 2.

Fig. 19. Harker diagram, $\mathrm{Zn}$ (in ppm). All other data as in Fig. 2.

Fig. 20. Harker diagram, $\mathrm{Zr}$ (in ppm). All other data as in Fig. 2. 
Table 1. Age constraints and lithology sumary, eastern Sierra Mevada mafic intrusions.

\begin{tabular}{|c|c|c|c|c|c|}
\hline Key & to Fig. 1 and Locality & Age & Constraint & Lithologies present & References \\
\hline 1 & Casa Diablo-Rock Creek & $210-148(2) \mathrm{my}$ & 1,4 & $\begin{array}{l}\text { ol gabbro, two-px-ol. gabbro, } \\
\text { cpx-hb gabbro, bio-hb diorite } \\
\text { bio-hb qtz diorite }\end{array}$ & $3,4,11,16$ \\
\hline 2. & Wheeler Crest & $210-148 \mathrm{my}$ & 1,4 & $\begin{array}{l}\text { cpx-hb gabbro, hb gabbro, } \\
\text { bia-hb gabbro }\end{array}$ & $1,3,4,16$ \\
\hline 3. & Pine Creek & $169 \mathrm{m.y}$. & U-Pb zírcon & bio-nb atz diorite & 1,14 \\
\hline 4. & Mt. Tom. & $>148$ my & 1,4 & $\begin{array}{l}\text { ho gabbro, ho diorite, } \\
\text { bio-hb atz diorite }\end{array}$ & $1,3,4,16$ \\
\hline 5. & Tungsten Hills & $150 \mathrm{my}$ & U-Pb zircon & $\begin{array}{l}c p x(h b) \text { gabbro, hb gabbro, } \\
\text { hb diorite }\end{array}$ & $1,4,14,16$ \\
\hline 6. & Bishop Creek-Habeggar's & $210-148 \mathrm{my}$ & 1,4 & $\begin{array}{l}\text { cpx-hb gabbro, ho gabbro, } \\
\text { bia-hb gabbro }\end{array}$ & $1,3,4,16$ \\
\hline 7. & Keough Hot Springs & $>148$ my & 1 & ol-cpx (hb) gabbro, hb gabbro & $1,14,16$ \\
\hline 8. & Shannon Canyon & $167-148 m y$ & 1,4 & $\begin{array}{l}\text { cpx (hb) gabbro, hb gabbro, } \\
\text { bio-hb diorite, atz diorite }\end{array}$ & $1,3,4,16$ \\
\hline 9. & McMurray Meadows & $150 \mathrm{my}$ & U-Pb zircon & $\begin{array}{l}\text { ol-cpx (hb) gabbro, hb gabbro, } \\
\text { bio-hb diorite }\end{array}$ & $1,14,16$ \\
\hline 10 & Armstrong Canyon & $154 \mathrm{my}$ & U-Pb zircon & $\begin{array}{l}\text { cpx-hb gabbro, hb gabbro, } \\
\text { bia-hb gabbro, atz diorite }\end{array}$ & $3,4,10,16$ \\
\hline 11. & Thidaut-Black Canyon & $165-148$ my & 1,4 & $\begin{array}{l}\text { cpx-hb gabbro, hb gabbro, } \\
\text { bio-hb gabbro, qtz diorite, } \\
\text { anorthosite }\end{array}$ & $3,4,10,16$ \\
\hline 12. & Baxter Lakes & $165(?)-148 \mathrm{my}$ & 1,4 & $\begin{array}{l}\text { cpx-hb gabbro, hb gabbro, } \\
\text { hb diorite }\end{array}$ & $3,4,10,16$ \\
\hline 13. & Wood Creek & $>148$ my & 1 & hb gabbro, hb diorite & $3,4,10,16$ \\
\hline \multicolumn{6}{|c|}{ Other Mafic Intrusions } \\
\hline & Mt. Morrison Pendant & Post-Late $P_{Z}$ & 6 & Hb diorite & 12 \\
\hline B. & Hidden Lakes & Late Cretaceous(?) & $2(?), 3,4$ & Hb gabbro,bio-hb diorite & $1,8,9,12,14$ \\
\hline c. & Pine Lake & 97.5 m.y. & U-Pb zircon & Hb diorite - qtz diorite & 8,15 \\
\hline ○. & Piute Pass-Lake Sabrina & Late Cretaceous & 4,5 & hb gabbro - mafic grd & $1,7,14$ \\
\hline E. & Black Giant & 147-81 m.y. (?) & 3,6 & hb gabbro & $2,4,6,13,14$ \\
\hline F. & Green Lake & Late Cretaceous(?) & $2(?), 5$ & hb gabbro - mafic grd & $1,7,15$ \\
\hline G. & Mt. Alice & Late Cretaceous(?) & $2(?), 4$ & hb diorite - qtz diorite & 1,15 \\
\hline H. & Palisade Creek-Upper Basin & Late Cretaceous & 4,5 & hb gabbro - mafic grd & $1,5,6,7,14$ \\
\hline J. & Onion Valley-Rae Lakes & Early Cretaceous(?) & 4,5 & Hb diorite - qtz diorite & 10,13 \\
\hline
\end{tabular}

Constraint code: 1) older than Independence dikes, 2) younger than Independence dikes, 3) older than surrounding dated granitoids, 4) younger than surrounding granitoids, 5) mutually cross-cutting relations and hybridization with surrounding plutons suggestive of similar age (Frost and Mahood, 1987), 6) intrusive into metamorphic rocks.

References: 1, Bateman, 1965; 2) Bateman and Moore, 1965; 3) Chen and Moore, 1979; 4) Chen and Moore, 1982; 5) Dodge and Moore, 1968; 6) Evernden and Kistler, 1970; 7) Frost and Mahood, 1987; 8) Frost and Mattinson, 19xx; 9) Lockwood and Lydon, 1975: 10) Moore, 1963; 11) Rinehart and Ross, 1957; 12) Rinenart and Ross, 1964; 13) Tobisch and others 1986; 14) Stern and others, 1981; 15) Frost, unpub. data; 16) this report. 
Table 2. Sample localities and descriptions, mafic rocks of the eastern Sierra Nevada.

Sample N. Lat W. Long Description

TUNGSTEN HILLS

$\begin{array}{lll}\text { TH-D } & 37.21^{\prime} 25^{\prime \prime} & 118.32^{\prime} 00^{\prime \prime} \\ \text { TH2-10 } & 37.21^{\prime} 20 & 118.31^{\prime} 50^{\prime \prime} \\ \text { TH2-16 } & 37.21^{\prime} 40^{\prime \prime} & 118.37^{\prime} 40^{\prime \prime} \\ \text { TH2-17 } & 37.21^{\prime} 40^{\prime \prime} & 118.31^{\prime} 40^{\prime \prime} \\ \text { TH2-20 } & 37.21^{\prime} 10^{\prime \prime} & 118.32^{\prime} 00^{\prime \prime} \\ \text { TH2-22 } & 37.21^{\prime} 15^{\prime \prime} & 118.31^{\prime} 40^{\prime \prime} \\ \text { TH2-23 } & 37.20^{\prime} 55^{\prime \prime} & 118.32^{\prime} 00^{\prime \prime} \\ \text { TH2-27 } & 37.20^{\prime} 00^{\prime \prime} & 118.32^{\prime} 00^{\prime \prime} \\ \text { TH2-29 } & 37.21^{\prime} 00^{\prime \prime} & 118.32^{\prime} 00^{\prime \prime} \\ \text { TH2-33 } & 37.21^{\prime} 00^{\prime \prime} & 118.32^{\prime} 00^{\prime \prime} \\ \text { TH2-36 } & 37.21^{\prime} 00 & 118.32^{\prime} 00^{\prime \prime}\end{array}$

CASA DIABLO

\begin{tabular}{lll}
\hline$C D 79-1 C$ & $37.35^{\prime} 00^{\prime \prime}$ & $118.40^{\prime} 00^{\prime \prime}$ \\
$C D-2$ & $37.35^{\prime} 00^{\prime \prime}$ & $118.40^{\prime \prime} 00^{\prime \prime}$ \\
$C D-4$ & $37.35^{\prime} 00^{\prime \prime}$ & $118.40^{\prime \prime} 00^{\prime \prime}$ \\
$C D-9$ & $37.35^{\prime} 00^{\prime \prime}$ & $118.40^{\prime \prime} 00^{\prime \prime}$ \\
& & \\
$C D-11$ & $37.35^{\prime} 00^{\prime \prime}$ & $118.40^{\prime \prime} 00^{\prime \prime}$ \\
$C D-13$ & $37.35^{\prime} 00^{\prime \prime}$ & $118.40^{\prime \prime} 00^{\prime \prime}$ \\
$C D-15$ & $37.35^{\prime} 00^{\prime \prime}$ & $118.40^{\prime \prime} 00^{\prime \prime}$ \\
$C D-17$ & $37.35^{\prime} 00^{\prime \prime}$ & $118.40^{\prime \prime} 00^{\prime \prime}$ \\
$C D-21$ & $37.35^{\prime} 00^{\prime \prime}$ & $118.40^{\prime \prime} 00^{\prime \prime}$
\end{tabular}

KEOUGH HOT SPRINGS

KHS-10A 37.15'05"

KHS-11A 37.15'05"

$118.23^{\prime} 40^{\prime \prime}$

$118.23^{\prime} 42^{\prime \prime}$

KHS-12

$37.15^{\prime} 10^{\prime \prime}$

$118.23^{\prime} 45^{\prime \prime}$

KHS-17

$37.15^{\prime} 40^{\prime \prime}$

KHS-19

$37.15^{\prime} 30^{\prime \prime}$

KHS-19B

$37.15^{\prime} 30^{\prime \prime}$

KHS-24

$37.15^{\prime} 25^{\prime \prime}$

KHS-33 $37.15^{\prime} 10^{\prime \prime}$ $118.23^{\prime} 03^{\prime \prime}$

$118.23^{\prime} 30^{\prime \prime}$

$118.23^{\prime} 30^{\prime \prime}$

$118.23^{\prime} 30^{\prime \prime}$

$118.22^{\prime} 40^{\prime \prime}$
Coarse, massive, poikilitic hornblende gabbro.

Fine, massive, hypidiomorphic hornblende diorite.

Fine, massive, hypidiomorphic hb diorite.

Vertically-layered, poikilitic hb gabbro.

Porphyritic hb gabbro.

Hypidiomorphic hb diorite of Tungsten Blue Mine.

Poikilitic, massive hb gabbro.

Hypidiomorphic, massive hornblende diorite.

Hypidiomorphic, coarse hornblende diorite.

Leucocratic, hypidiomorphic hornblende diorite.

Fine grained, leucocratic microgabbro.

Layered, biotite-rich, hornblende gabbro.

Cumulus ol-cpx-hb gabbro. 01 altered to iddingsite. Layered cumulus ol-cpx-hb gabbro.

Coarse ol-cpx-hb gabbro. Kelyphitic hb-pl rims on 01 .

Medium-grained hypidiomorphic hornblende gabbro.

Fine-grained bio-hb quartz diorite.

Coarse hornblende gabbro.

Coarse hornblende gabbro. Hb is euhdral, randomly oriented, cored by aggregate of $\mathrm{pl}+\mathrm{hb}$.

Coarse ol-cpx-hb gabbro. 01 is rimmed by radial growths of $p l+c p x+p l$.

THIBAUT-BLACK CANYON

SM-1C 36.52"10" 118.20'30"

SM-3 $\quad 36.51^{\prime} 05^{\prime \prime} \quad 118.20^{\prime} 25^{\prime \prime}$

SM-7A 36.52'00" $118.20^{\prime} 10^{\prime \prime}$

SM-7B $36.52^{\prime} 00^{\prime \prime} 118.20^{\prime} 10^{\prime \prime}$

SM-7C $\quad 36.52^{\prime} 00^{\prime \prime} \quad 118.20^{\prime} 10^{\prime \prime}$

SM-8 $36.52^{\prime} 00^{\prime \prime} \quad 118.20^{\prime} 10^{\prime \prime}$

Massive biotite-hornblende diorite.

Coarse lens of hornblende gabbro in finer hb diorite.

Faintly layered hypidiomorphic hornblende gabbro. Homogeneous hornblende gabbro.

Coarse, homogeneous hornblende gabbro.

Mafic hornblende gabbro in gradational contact with KHS 1-19.

Coarse, felsic homogeneous hornblende gabbro.

Layered hornblende gabbro.

Layered, hypidiomorphic biotite-hornblende

granodiorite.

Hornblende-plagioclase pegmatite.

Homogeneous, felsic biotite-hornblende diorite

Anorthosite (7\% hornblendetepidote, remainder is

euhedral plagioclase in random orientation, up to $4 \mathrm{~mm}$ ).

Anorthosite similar to SM-7B.

Anorthosite, color index 3 (hbtepidote). Euhedral pl to $5 \mathrm{~mm}$ in random orientation. 
Table 2. Continued.

\begin{tabular}{|c|c|c|c|}
\hline SM-9 & $36.52^{\prime} 00^{\prime \prime}$ & $118.20^{\prime} 10^{\prime \prime}$ & Anorthosite, color index 3 (hb+epidote). Euhedral \\
\hline SM-10 & $36.52^{\prime} 05^{\prime \prime}$ & $118.20^{\prime} 15^{\prime \prime}$ & $\begin{array}{l}\text { Biotite-hornblende gabbro, sharply cut by dikes of } \\
\text { SM-9 type rock. }\end{array}$ \\
\hline $\begin{array}{l}\text { SM-16 } \\
\text { SM-19 }\end{array}$ & $\begin{array}{l}36.52^{\prime} 20^{\prime \prime} \\
36.52^{\prime} 20^{\prime \prime}\end{array}$ & $\begin{array}{l}118.17^{\prime} 30^{\prime \prime} \\
118.17^{\prime \prime} 35^{\prime \prime}\end{array}$ & $\begin{array}{l}\text { Biotite-hornblende mafic granodiorite. } \\
\text { Biotite-hornblende quartz diorite. }\end{array}$ \\
\hline
\end{tabular}

ARMSTRONG CANYON

\begin{tabular}{lll}
\hline$A C-8 A$ & $36.56^{\prime} 10^{\prime \prime}$ & $118.20^{\prime} 30^{\prime \prime}$ \\
$A C-9$ & $36.56^{\prime} 15^{\prime \prime}$ & $118.20^{\prime} 30^{\prime \prime}$ \\
$A C-21 A$ & $36.57^{\prime} 35^{\prime \prime}$ & $118.20^{\prime} 30^{\prime \prime}$ \\
& & \\
$A C-21 C$ & $36.57^{\prime} 35^{\prime \prime}$ & $118.20^{\prime} 30^{\prime \prime}$ \\
$A C-21 D$ & $36.57^{\prime} 35^{\prime \prime}$ & $118.20^{\prime} 30^{\prime \prime}$ \\
AC-23 & $36.57^{\prime} 25^{\prime \prime}$ & $118.20^{\prime} 50^{\prime \prime}$ \\
& & \\
AC-26B & $36.57^{\prime} 20^{\prime \prime}$ & $118.21^{\prime} 20^{\prime \prime}$ \\
& & \\
AC-27 & $36.57^{\prime} 15^{\prime \prime}$ & $118.21^{\prime} 20^{\prime \prime}$ \\
AC-29 & $36.57^{\prime} 00^{\prime \prime}$ & $118.22^{\prime} 00^{\prime \prime}$
\end{tabular}

Coarse hornblende gabbro.

Coarse hornblende diorite.

Coarse, mafic, hornblende gabbro at contact with younger Goodale pluton of Moore (1963).

Coarse, "spotted" (poikilitic) hornblende gabbro. Crudely-layered biotite-hornblende gabbro.

Faintly-layered biotite-hornblende, medium grained diorite.

Coarse, biotite-hornblende quartz diorite. Dating sample from Armstrong Canyon is similar to this sample.

Fine-grained homogeneous hornblende gabbro.

Homogeneous, hypidiomorphic biotite-hornblende diorite.

MT. TOM

\begin{tabular}{lll}
\hline$T-3$ & $37.20^{\prime} 30^{\prime \prime}$ & $118.38^{\prime} 30^{\prime \prime}$ \\
$T-5$ & $37.20^{\prime} 30^{\prime \prime}$ & $118.38^{\prime} 30^{\prime \prime}$ \\
$T-7$ & $37.20^{\prime} 30^{\prime \prime}$ & $118.38^{\prime} 30^{\prime \prime}$ \\
$T-10$ & $37.20^{\prime} 30^{\prime \prime}$ & $118.38^{\prime} 30^{\prime \prime}$
\end{tabular}

Massive, medium-grained, hornblende diorite. Massive, medium-grained, hornblende gabbro. Massive, medium-grained, hornblende diorite. Massive, medium-grained, hornblende diorite.

\section{MCMURRY MEADOWS}

\begin{tabular}{|c|c|c|}
\hline$=M$ & $40^{\prime \prime}$ & $118.21^{\prime} 05^{\prime \prime}$ \\
\hline $\begin{array}{l}M-1 E \\
M-7 A\end{array}$ & & \\
\hline :M- & $05^{\prime \prime}$ & יברי \\
\hline$M$ & r & r \\
\hline 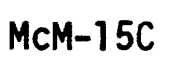 & & \\
\hline cM-16 & $37.04^{\prime} 05^{\prime \prime}$ & \\
\hline $\begin{array}{l}C M \\
=M \\
=M\end{array}$ & $\begin{array}{l}' 05 \text { ' } \\
\text { '05" } \\
\text { ' } 35 \text { " } \\
\text { '00" }\end{array}$ & $\begin{array}{l}11 \\
11 \\
11\end{array}$ \\
\hline$=M-3$ & $\begin{array}{l}7^{\prime} 00^{\prime \prime} \\
t^{\prime} 30^{\prime \prime} \\
t^{\prime} 30^{\prime \prime}\end{array}$ & 40 \\
\hline
\end{tabular}

Homogeneous, medium grained hornblende gabbro. Coarse, cumulus hb gabbro. Original cpx altered to hornblende.

Poikilitic, mafic, hornblende gabbro.

Poikilitic, mafic, horizontal-layered, coarse hornblende gabbro.

Poikilitic, mafic, horizontal-layered, coarse hornblende gabbro.

Felsic, faintly horizontal-layered, coarse, poikilitic hornblende gabbro.

Plagioclase-rich layer in horizontal-layered hb gabbro.

Mafic layer in horizontal-layered poikilitic hornblende gabbro.

Homogeneous, poikilitic hornblende gabbro.

Homogeneous, poikilitic hornblende gabbro.

Coarse, felsic hornblende gabbro.

Coarse, porphyritic hornblende gabbro. Faint horizontal layering visible locally.

Well-layered hornblende gabbro. Mafic facies.

Felsic layer in hornblende gabbro.

Mafic hornblende gabbro.

Mafic hornblende gabbro. 
Table 3. Major element determinations.

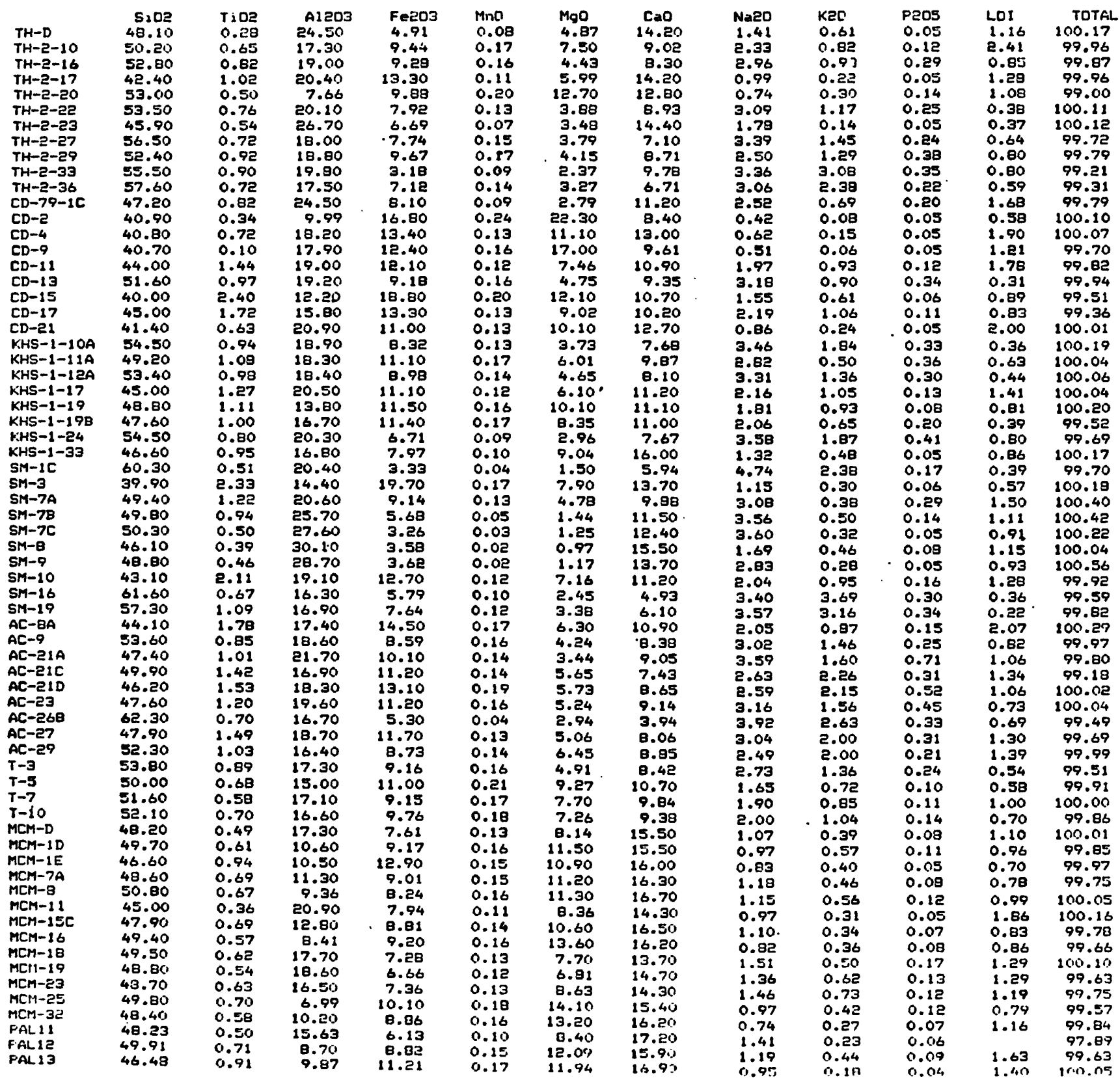


Table 4. Trace element determinations. Reported values in ppm.

\begin{tabular}{|c|c|c|c|c|c|c|c|c|c|c|}
\hline & v & $C R$ & NI & $\mathrm{ZN}$ & RB & SR & $Y$ & $2 R$ & NB & $B A$ \\
\hline $\begin{array}{l}T H-D \\
T H-2-10\end{array}$ & 100 & 110 & 20 & 46 & & 635 & 13 & 41 & 3 & 240 \\
\hline $\mathrm{TH}-\mathrm{C}-10$ & 230 & 0 & 50 & 90 & 33 & 500 & 15 & 54 & 2 & 540 \\
\hline$T H-2-16$ & 195 & 28 & 16 & 110 & 23 & 620 & 17 & 65 & 6 & 625 \\
\hline TH-E-17 & 410 & 0 & 17 & 69 & 14 & 620 & 10 & 34 & 2 & 170 \\
\hline $\mathrm{TH}-\mathrm{E}-20$ & 250 & 0 & 160 & 125 & 11 & 270 & 13 & 48 & 2 & 280 \\
\hline TH-Z-2E & 175 & 29 & 19 & 81 & 23 & 575 & e2 & B1 & 5 & 630 \\
\hline $\mathrm{TH}-2-23$ & 260 & 0 & 29 & 43 & 14 & 780 & 7 & 32 & 2 & 140 \\
\hline$T H-2-27$ & 170 & 0 & 23 & 97 & 38 & seo & 20 & 145 & 2 & 1000 \\
\hline TH-2-29 & 230 & 28 & 13 & 110 & 41 & 600 & 23 & 88 & 7 & 720 \\
\hline$T+1-2-33$ & 0 & 0 & 13 & 83 & 93 & 625 & 20 & 60 & 2 & 5000 \\
\hline$T H-2-36$ & $160^{\circ}$ & 0 & 23 & 100 & 58 & 450 & 24 & 140 & 2 & 1500 \\
\hline$C D-79-1 C$ & 2010 & 0 & 10 & 60 & 34 & 855 & 16 & 59 & 2 & 480 \\
\hline$C D-2$ & 140 & 365 & 165 & 115 & 11 & 305 & 6 & 19 & $\bar{z}$ & AS \\
\hline CI -4 & 325 & 140 & 66 & 89 & 11 & 590 & 6 & 29 & 2 & 120 \\
\hline CD-9 & 60 & 360 & 125 & 99 & 11 & 620 & 4 & 25 & 2 & 77 \\
\hline$C[-1]$ & 400 & 72 & 25 & 92 & 31 & 700 & 16 & 58 & 4 & 445 \\
\hline$C D-1]$ & 210 & 40 & 21 & 114 & 29 & 821 & 15 & 95 & 5 & 800 \\
\hline$c n-15$ & 625 & 260 & 52 & 135 & 17 & 260 & 23 & 59 & 2 & 250 \\
\hline$C D-17$ & 450 & 110 & 35 & 90 & 25 & 525 & 18 & 65 & 7 & 490 \\
\hline$C D-21$ & 225 & 115 & 66 & 93 & 12 & 790 & 7 & 30 & 3 & 125 \\
\hline$H+13-1-10 \mathrm{~A}$ & 175 & 10 & 20 & 110 & 55 & 740 & 19 & 125 & $B$ & 1080 \\
\hline $1 H 5-1-11 A$ & 260 & 45 & 26 & 140 & 18 & 850 & 15 & 59 & 5 & 505 \\
\hline $1 \mathrm{HS}-1-12 \mathrm{~A}$ & 210 & 46 & 25 & 100 & 44 & 720 & 17 & 110 & 8 & 810 \\
\hline $1: 13-1-17$ & 370 & 0 & 18 & 105 & 34 & 670 & 19 & 66 & 2 & 355 \\
\hline HS-1-19 & 265 & 0 & 89 & 115 & 34 & 450 & 14 & 61 & $\overline{2}$ & 1040 \\
\hline $1 H 5-1-198$ & 320 & 110 & 57 & 120 & 36 & 750 & 11 & 50 & 5 & 355 \\
\hline $1.45-1-24$ & 150 & 0 & 10 & 95 & 72 & 780 & 17 & 125 & 2 & 1030 \\
\hline KHS - - - -33 & 250 & 0 & 86 & 61 & 13 & 600 & 13 & 54 & 2 & 220 \\
\hline$S M-1 C$ & 73 & 0 & 10 & 43 & 71 & 750 & 16 & 170 & $\overline{2}$ & 820 \\
\hline $5 M-3$ & 610 & 0 & 12 & 120 & 14 & $\mathbf{5 7 0}$ & 12 & 49 & 2 & 215 \\
\hline $511-7 A$ & 245 & 44 & 25 & 110 & 15 & 1020 & 9 & 40 & 4 & 495 \\
\hline $5 M-7 E$ & 150 & 5 & 11 & 45 & 20 & 1250 & 11 & 60 & 4 & 600 \\
\hline $314-7 C$ & 97 & 5 & 13 & 30 & 17 & 1270 & 12 & 44 & 3 & 525 \\
\hline $5 i 1-3$ & Bo & 5 & 8 & 22 & 21 & 1370 & 12 & 61 & 3 & 275 \\
\hline$S M-9$ & 95 & 5 & 9 & 21 & 15 & 1100 & 11 & 51 & 4 & 370 \\
\hline $511-10$ & 470 & 90 & 57 & 69 & 30 & 800 & 16 & 69 & 6 & 365 \\
\hline $5 M-16$ & 115 & 10 & 16 & 55 & 100 & 635 & 22 & 134 & 12 & 1150 \\
\hline $511-19$ & 160 & 44 & 36 & 84 & 125 & 540 & 25 & 180 & 12 & 780 \\
\hline GIC-BA & 550 & 45 & 17 & 82 & 45 & 450 & 19 & 84 & 5 & 270 \\
\hline$A C-9$ & 210 & 38 & 21 & B6 & 40 & 475 & 22 & 110 & 7 & 670 \\
\hline$A C-21 A$ & 135 & 0 & 13 & 145 & 64 & 1000 & 23 & 205 & 2 & 1130 \\
\hline$G C-21 C$ & 290 & 0 & 38 & 120 & 70 & 880 & 23 & 175 & 2 & 1210 \\
\hline$A C-21 D$ & 290 & 0 & 29 & 155 & 60 & 860 & 25 & 175 & 2 & 1250 \\
\hline$f C-23$ & 220 & 39 & 25 & 130 & 49 & 830 & 25 & 185 & 11 & 530 \\
\hline$G+C-26 B$ & 125 & 11 & 14 & 35 & 122 & 622 & 5อ & 150 & 11 & 1000 \\
\hline$+C-27$ & 285 & 0 & 45 & 148 & 60 & 720 & 21 & 170 & 2 & 1120 \\
\hline$A C-29$ & 270 & 0 & 40 & 105 & 64 & 540 & $2 i$ & 110 & 2 & 750 \\
\hline$T-3$ & 240 & 70 & 21 & 94 & 42 & 500 & 19 & 74 & 8 & 750 \\
\hline$T-5$ & 252 & 150 & 42 & 100 & 29 & 430 & 14 & 45 & 5 & 350 \\
\hline$T-7$ & 210 & 120 & 34 & 84 & 31 & 495 & 12 & 63 & 5 & 480 \\
\hline$T-10$ & 245 & 105 & 34 & 100 & 38 & 480 & 17 & 69 & 4 & 560 \\
\hline$M C H-D$ & 185 & 65 & 77 & 62 & 12 & 700 & 12 & 50 & 4 & 165 \\
\hline IEN-1D & 235 & 740 & 140 & 64 & 27 & 300 & 14 & 45 & 6 & 210 \\
\hline IACM-1E & 485 & 500 & 78 & 63 & 12 & 320 & 12 & 42 & 3 & 160 \\
\hline IICM-7A & & 460 & 110 & 60 & 14 & 350 & 14 & 53 & 5 & 150 \\
\hline MEM-G & 265 & 900 & 133 & 44 & 16 & 280 & 14 & 64 & 3 & 170 \\
\hline$M C 11-11$ & 150 & 40 & 86 & 53 & 16 & 650 & 日 & 39 & 3 & 110 \\
\hline MCM-15C & 265 & 370 & $\cdot 74$ & 46 & 12 & 360 & 12 & 43 & 4 & $1 \in S$ \\
\hline MCM-16 & 245 & 310 & 190 & 59 & 12 & 230 & 13 & 44 & 3 & 165 \\
\hline HCri-1B & 215 & 180 & 61 & 62 & 13 & 535 & $\begin{array}{l}20 \\
12\end{array}$ & 54 & 5 & 275 \\
\hline$M C M-19$ & 195 & 60 & 69 & 58 & 20 & 575 & 14 & 56 & 3 & 270 \\
\hline $11 C n-23$ & 200 & 180 & 84 & 60 & 27 & 500 & 14 & 60 & 4 & 230 \\
\hline$M=M-25$ & 265 & 370 & 185 & 65 & $\begin{array}{l}27 \\
11\end{array}$ & $\begin{array}{l}500 \\
200\end{array}$ & $\begin{array}{l}14 \\
15\end{array}$ & 48 & 3 & 225 \\
\hline$M C M-3 E$ & 220 & 250 & 155 & 57 & 9 & & & 43 & 3 & 120 \\
\hline FALL11 & 0 & 173 & 123 & 38 & 5 & 494 & 10 & 35 & 1 & 0 \\
\hline FAL 12 & 0 & 274 & 140 & 59 & 10 & 225 & 16 & 48 & 3 & 0 \\
\hline FitL 13 & 0 & 277 & 118 & 70 & 4 & 295 & 14 & 33 & 1 & 96 \\
\hline
\end{tabular}


Table $\$$ U-Pb zircon ages, eastern Sierra Nevada mafic plutonic rocks.

\begin{tabular}{|c|c|c|c|c|c|c|c|c|}
\hline $\begin{array}{l}\text { trusion } \\
\text { mber and } \\
\text { mple }\end{array}$ & $\begin{array}{r}U^{238} \\
\text { (ppm) }\end{array}$ & $\begin{array}{l}\mathrm{Pb}^{206^{*}} \\
(\mathrm{ppm})\end{array}$ & $\frac{\mathrm{Pb}^{2081}}{\mathrm{~Pb}^{206}}$ & $\frac{\mathrm{Pb}^{2071}}{\mathrm{~Pb}^{206}}$ & $\frac{\mathrm{Pb}^{2041}}{\mathrm{~Pb}^{206}}$ & $\frac{\mathrm{Pb}^{206^{\star 2}}}{u^{238}}$ & $\frac{\mathrm{Pb}^{207^{\star 2}}}{u^{235}}$ & $\mathrm{~Pb}^{206}$ \\
\hline
\end{tabular}

Mafic intrusions

$\begin{array}{llrlllllll}5 & \text { TH-D } & 384.3 & 7.846 & 0.2363 & 0.05150 & 0.0001596 & 150.3 & 150.6 & 155 \pm 8 \\ 9 & \text { MCM-D } & 1397.1 & 28.64 & 0.3968 & 0.05035 & 0.0000842 & 150.8 & 151.0 & 154 \pm 7 \\ 10 & \text { AC } & 573.9 & 12.00 & 0.2044 & 0.05297 & 0.000246 & 153.8 & 154.5 & 165+10\end{array}$

Tungsten Hills Granite

THG $\quad 1658.0 \quad 41.12$

$\begin{array}{llllllll}1658.0 & 41.12 & 0.1031 & 0.05068 & 0.0000477 & 182.0 & 182.8 & 194+4\end{array}$

* Radiogenic lead. 1 Isotopic ratios corrected for fractionation. 2 Age in $10^{6}$ years. Uncertainty in $206 \star / 238$ and $207 \star / 235$ ages are 1 per cent. 
FIGURE CAPTIONS

Fig. 1. Location and regional geologic map. Sources: Rinehart and Ross, 1957, 1964; Moore, 1963; Bateman, 1965; Bateman and Moore, 1965; Moore and Foster, 1980; Stern et al, 1981; Chen and Moore, 1979, 1982; Frost and Mahood, 1987; Frost and Mattinson, in prep; Tobisch et al, 1986)

Fig. 2. Harker diagram, Ti02, in weight percent. All data recalculated anhydrous. Locality coding: A - Armstrong Canyon; C - Casa Diablo-Rock Creek; K - Keough Hot Springs; M - McMurry Meadows, felsic layers; Z - McMurry Meadows, mafic layers; S - Thibaut-Black Canyon; T - Tungsten Hills; X Mt. Tom.

Fig. 3. Harker diagram, Al203. All data as in Fig. 2.

Fig. 4. Harker diagram, Fe0* (All iron recalculated as FeO). All data as in Fig. 2 .

Fig. 5. Harker diagram, MgO. All data as in Fig. 2.

Fig. 6. Harker diagram, MnO. All data as in Fig. 2.

Fig. 7. Harker diagram, CaO. All data as in Fig. 2.

Fig. 8. Harker diagram, Na20. All data as in Fig. 2.

Fig. 9. Harker diagram, K20. All data as in Fig. 2.

Fig. 10. Harker diagram, P205. All data as in Fig. 2.

Fig. 11. Harker diagram, Ba (in ppm). All other data as in Fig. 2.

Fig. 12. Harker diagram, $\mathrm{Cr}$ (in ppm). Samples shown as zero did not have $\mathrm{Cr}$ determined. All other data as in Fig. 2 .

Fig. 13. Harker diagram, Nb (in ppm). All other data as in Fig. 2.

Fig. 14. Harker diagram, $\mathrm{Ni}$ (in ppm). All other data as in Fig. 2.

Fig. 15. Harker diagram, Rb (in ppm). All other data as in Fig. 2.

Fig. 16. Harker diagram, $S r($ in ppm). All other data as in Fig. 2 .

Fig. 17. Harker diagram, V (in ppm). All other data as in Fig. 2.

Fig. 18. Harker diagram, Y (in ppm). All other data as in Fig. 2.

Fig. 19. Harker diagram, Zn (in ppm). All other data as in Fig. 2.

Fig. 20. Harker diagram, $\mathrm{Zr}$ (in ppm). All other data as in Fig. 2 . 


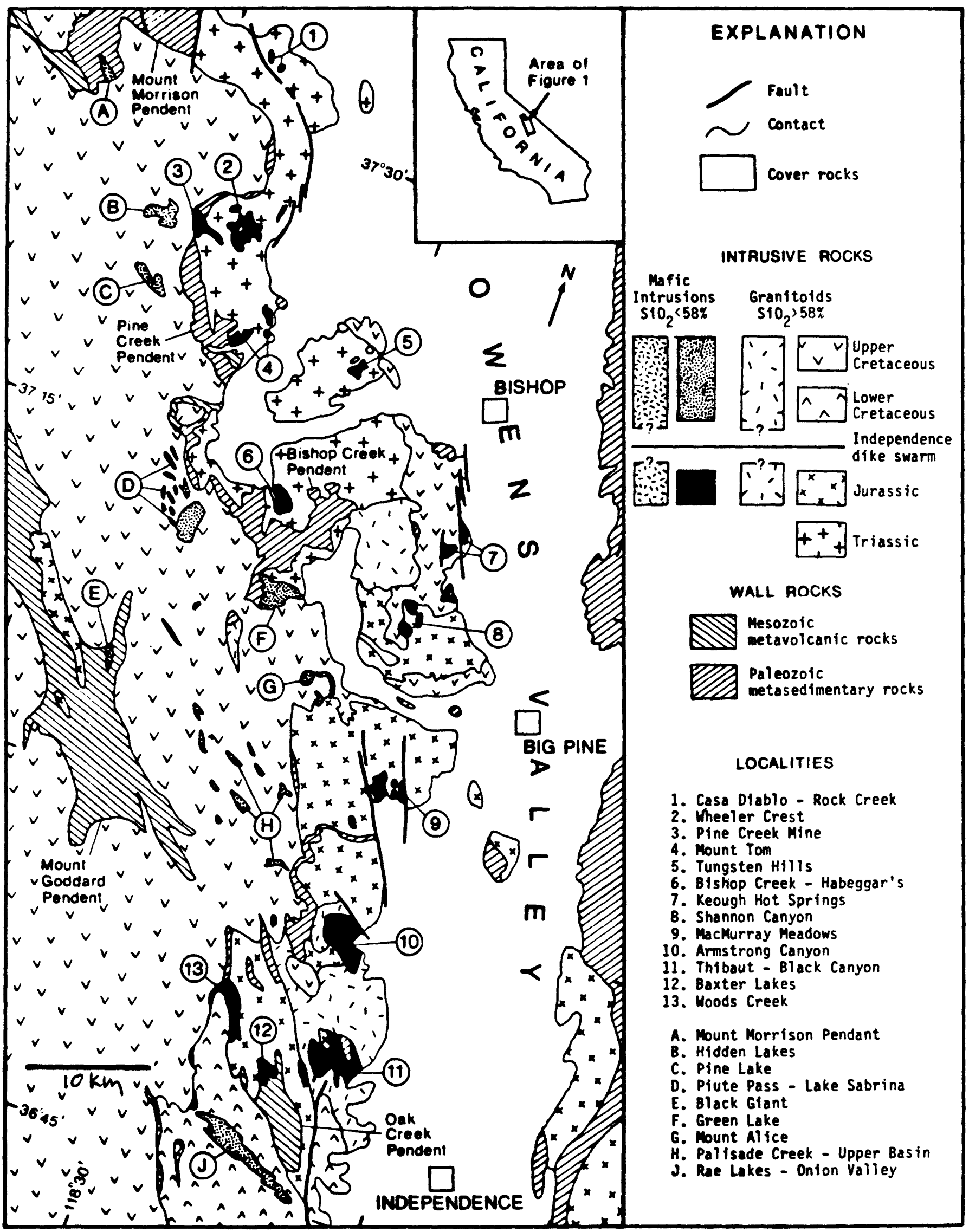

ñ. 1 


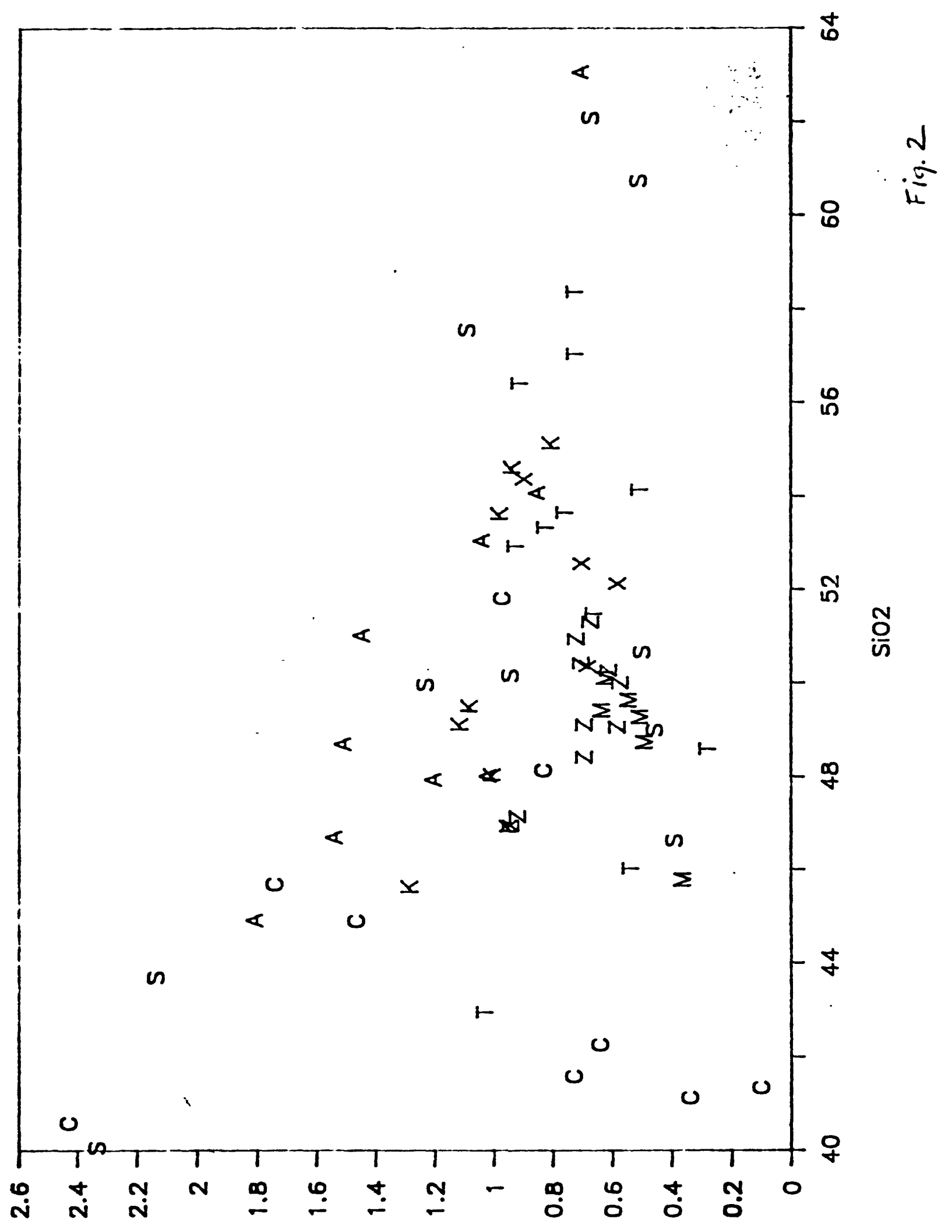

2011 


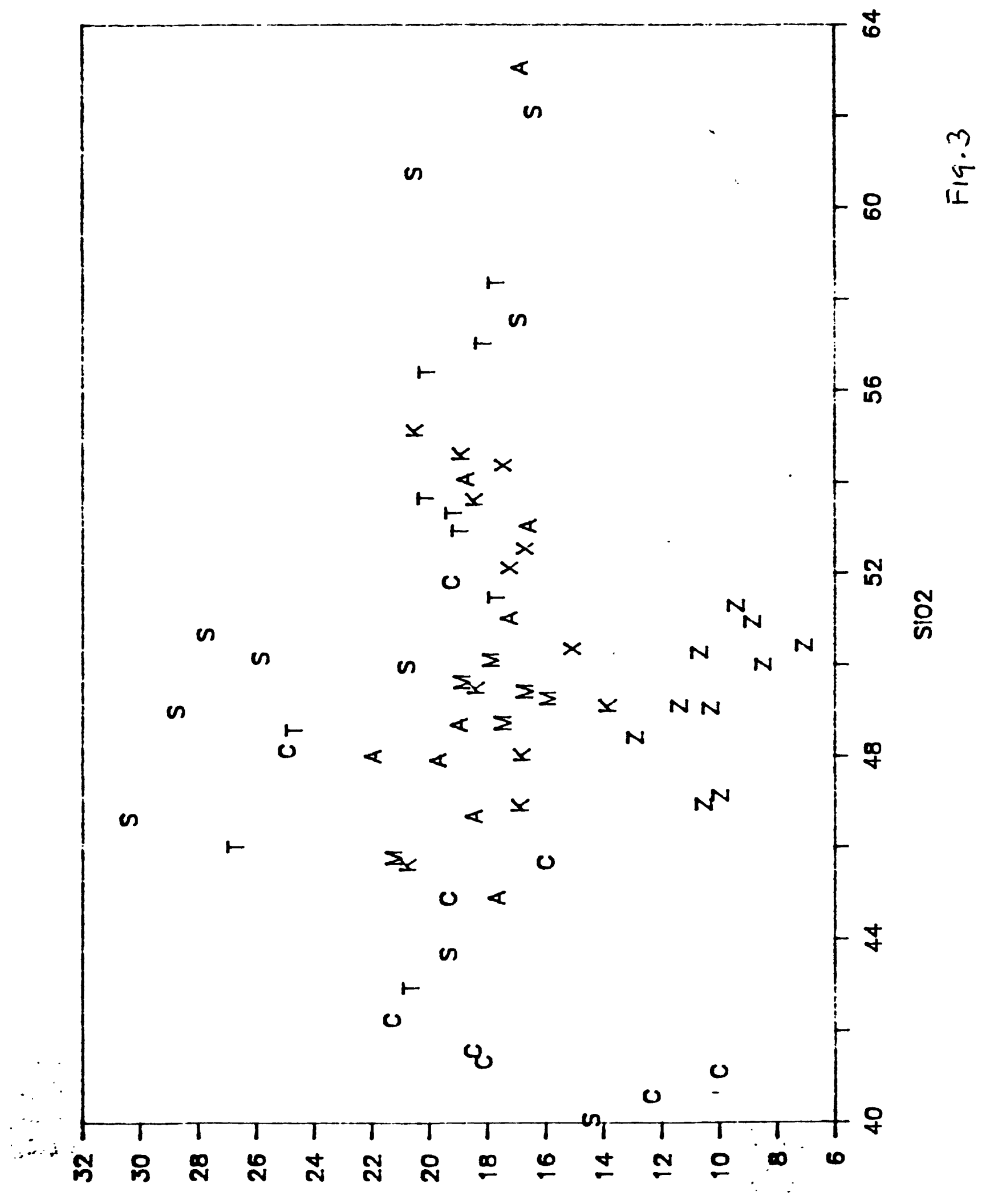

EOzit 


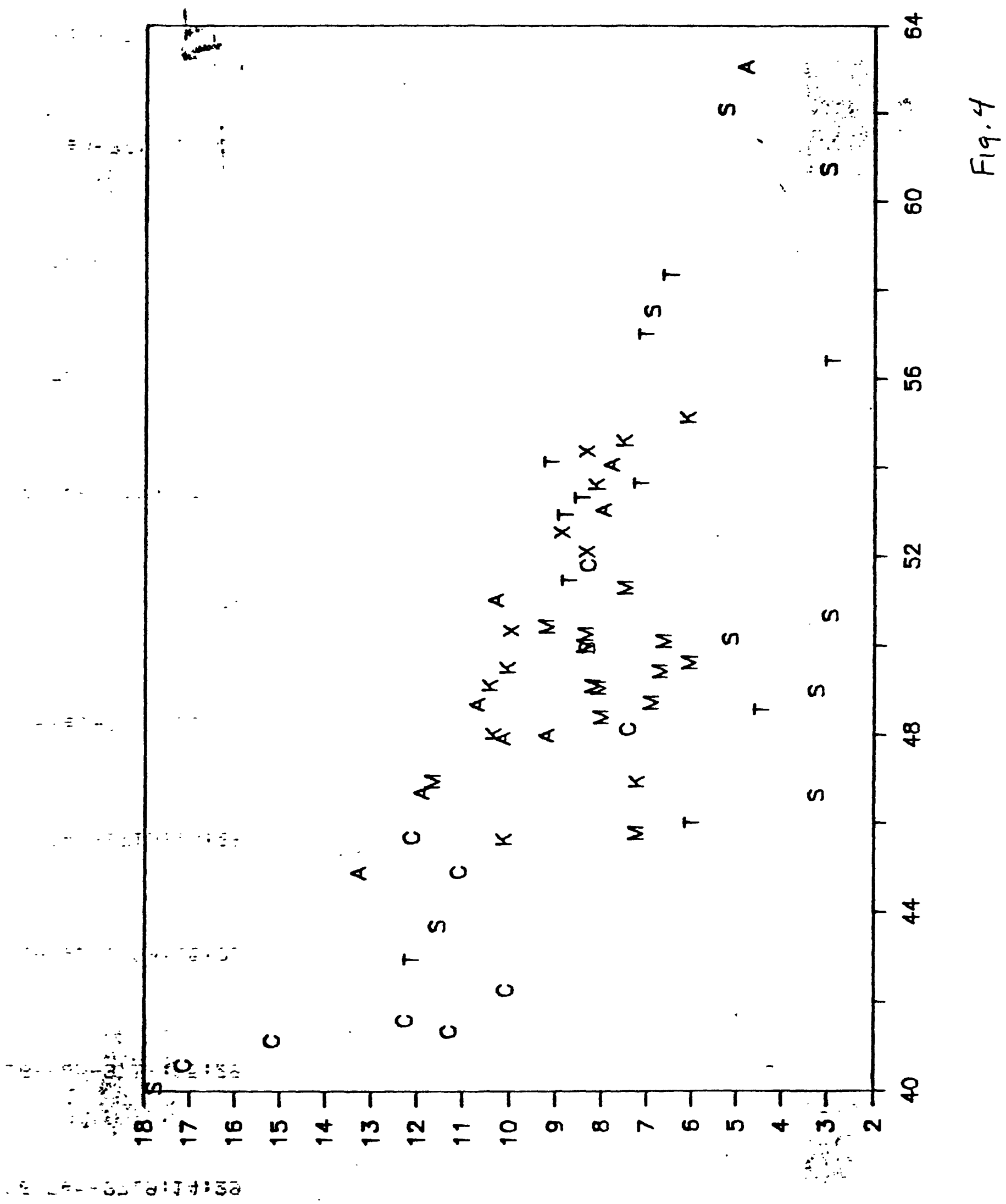




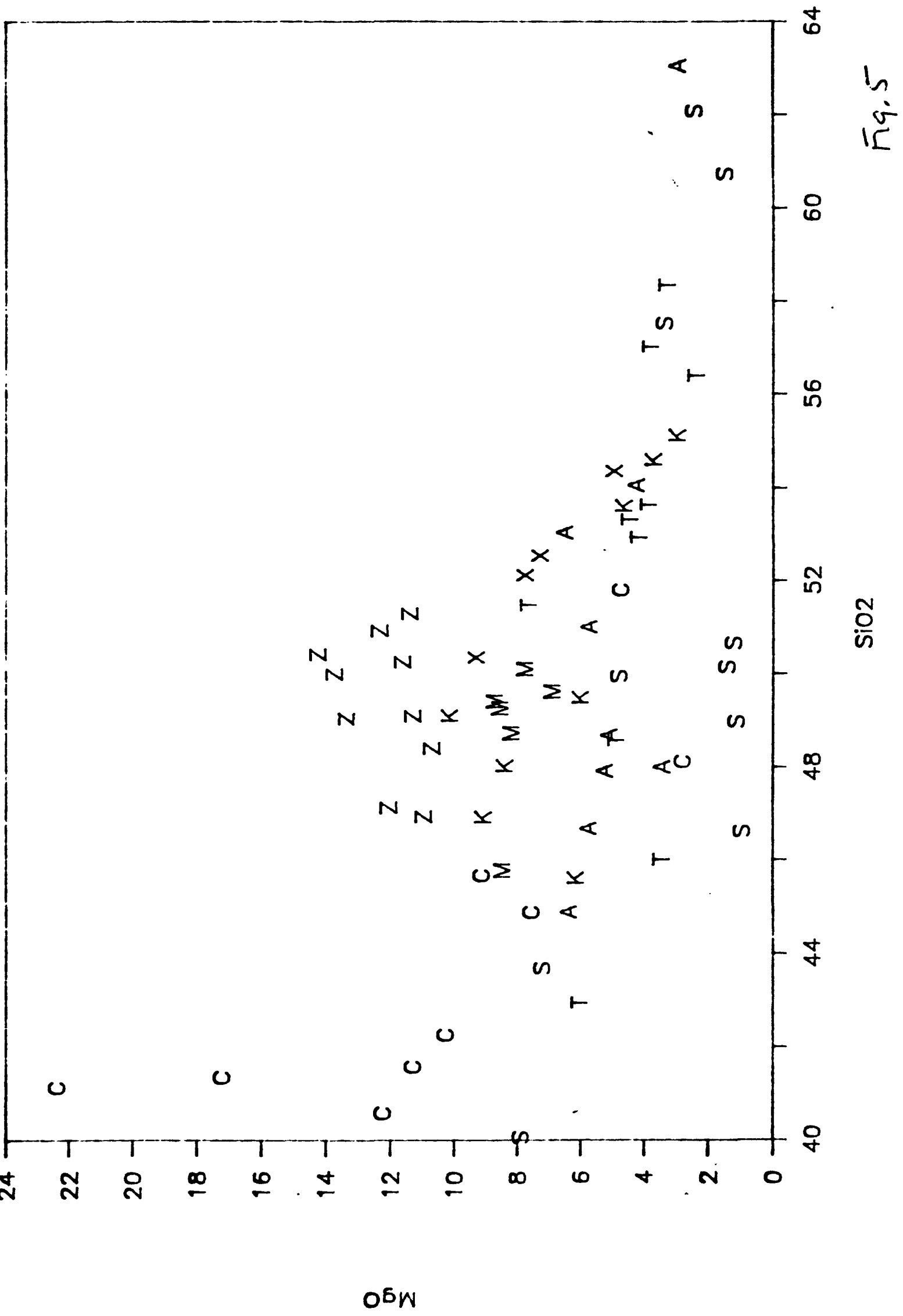

17 


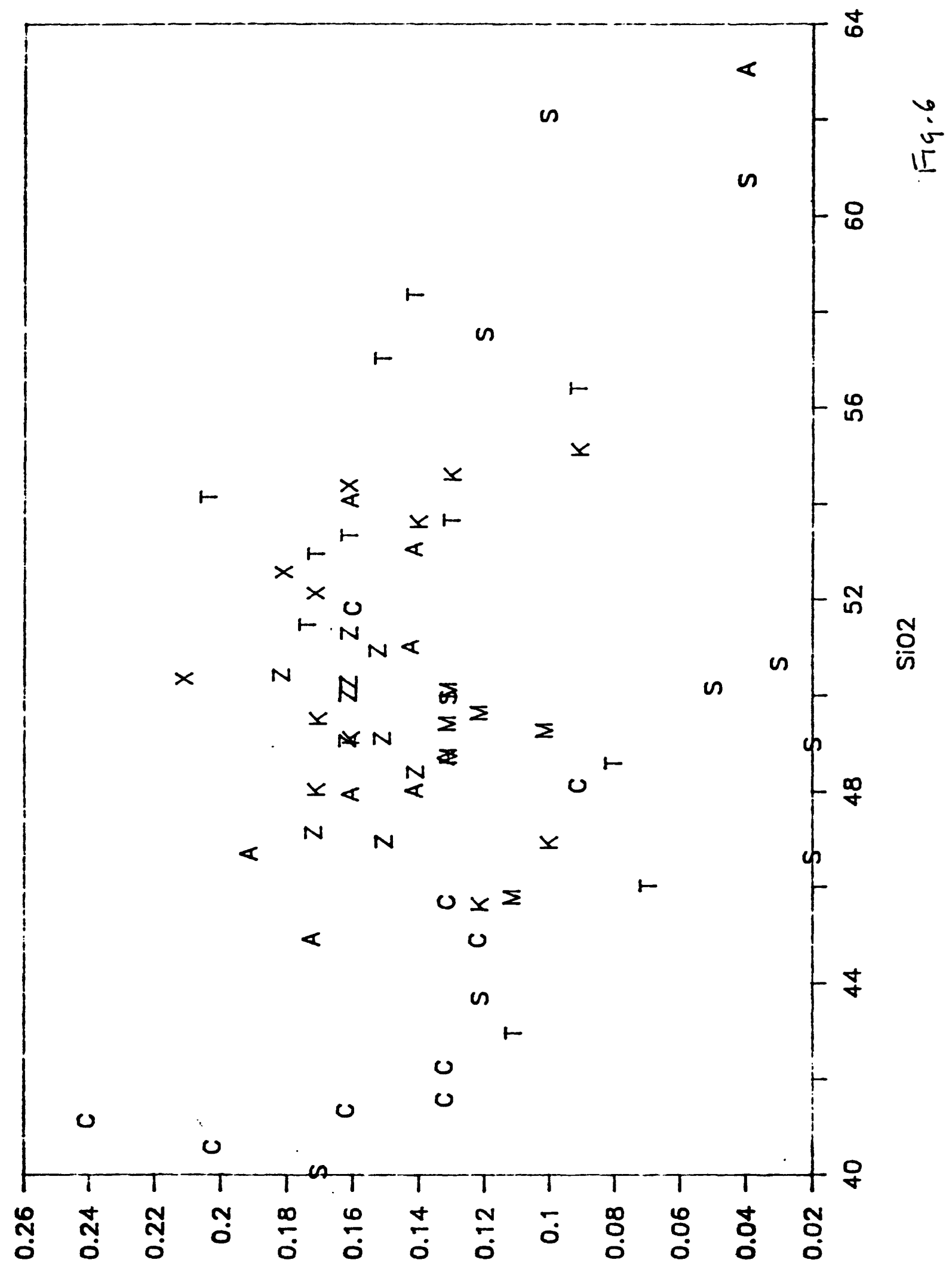

oun 


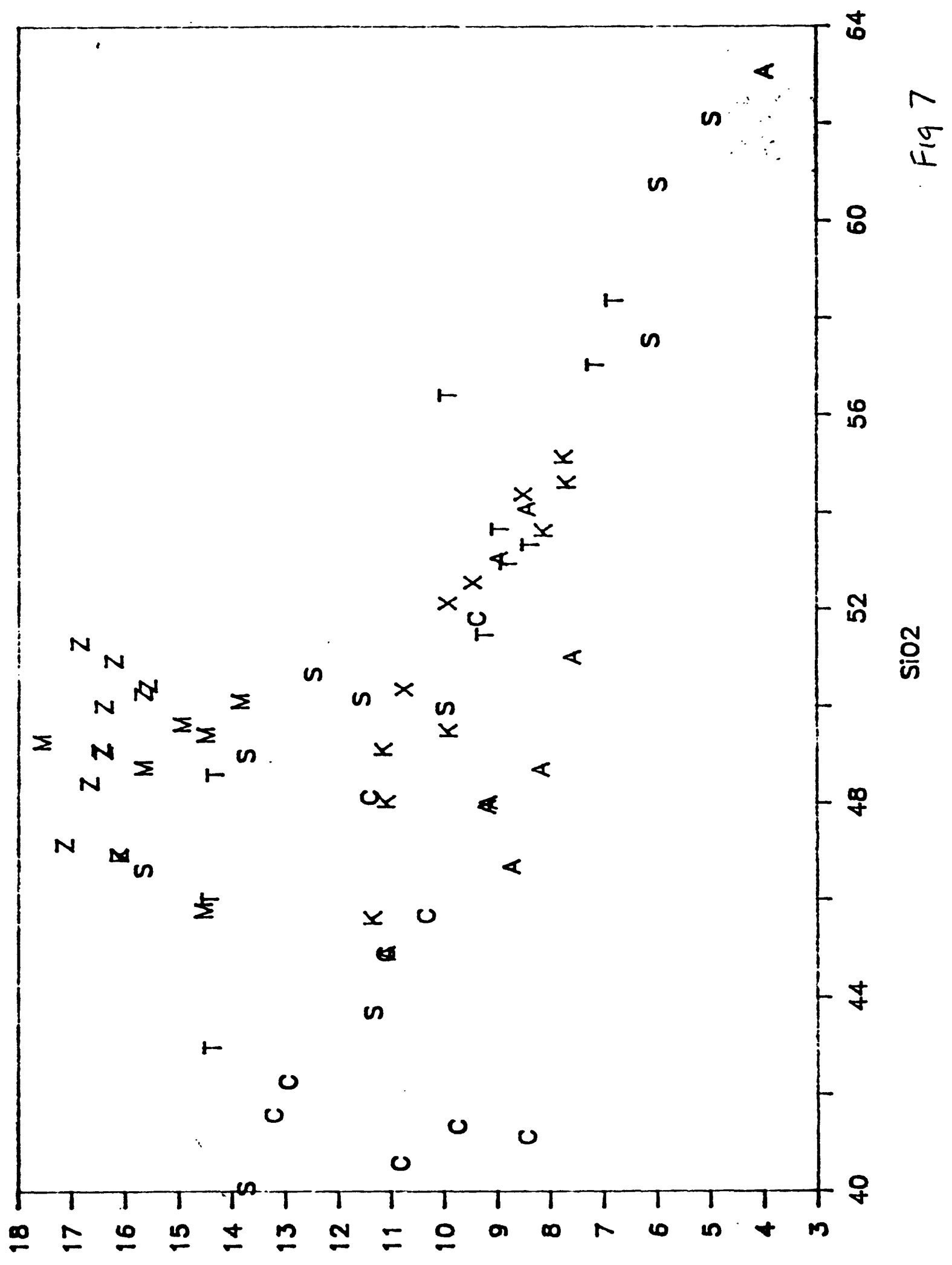

000 


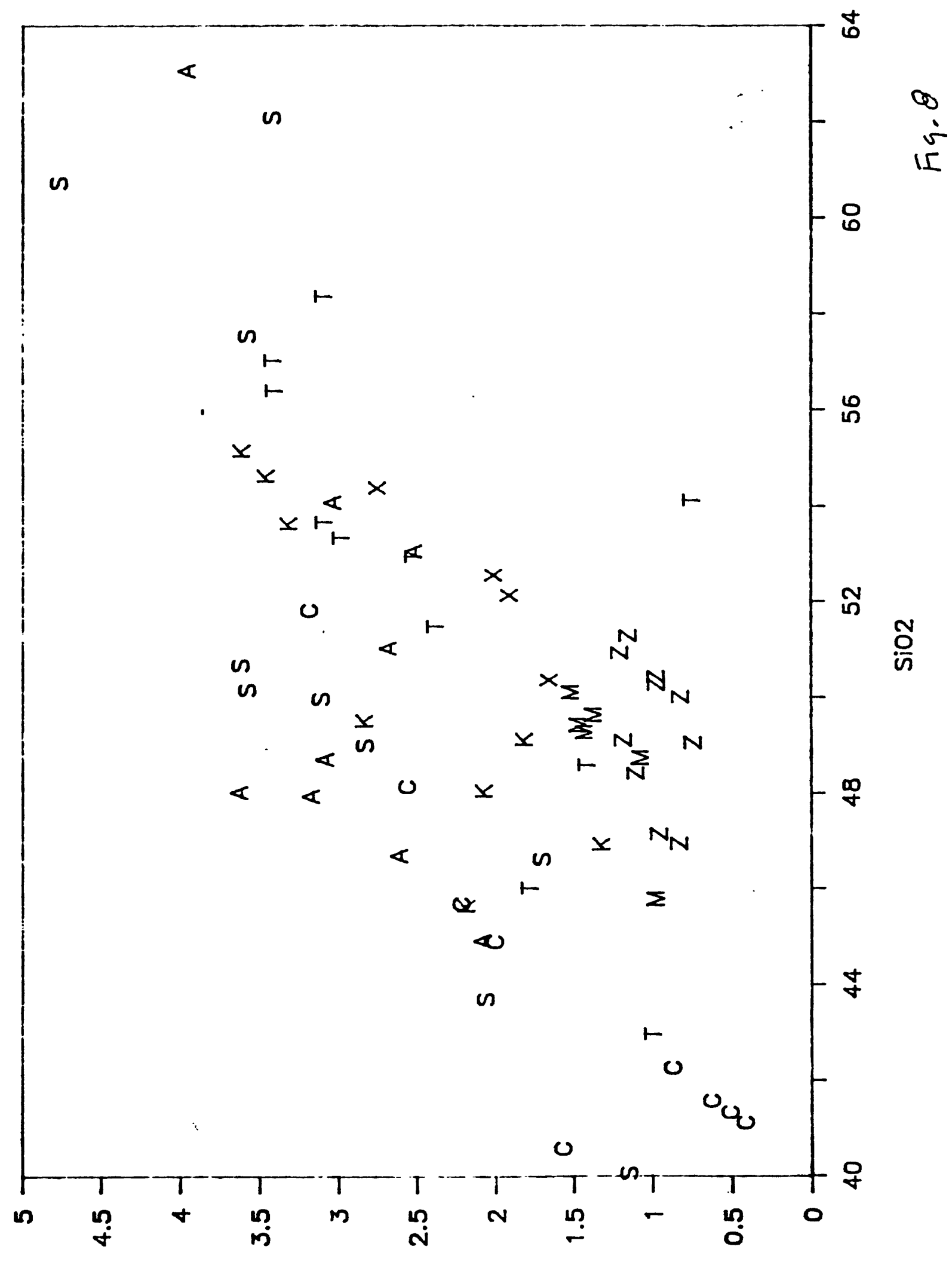

OZDN 


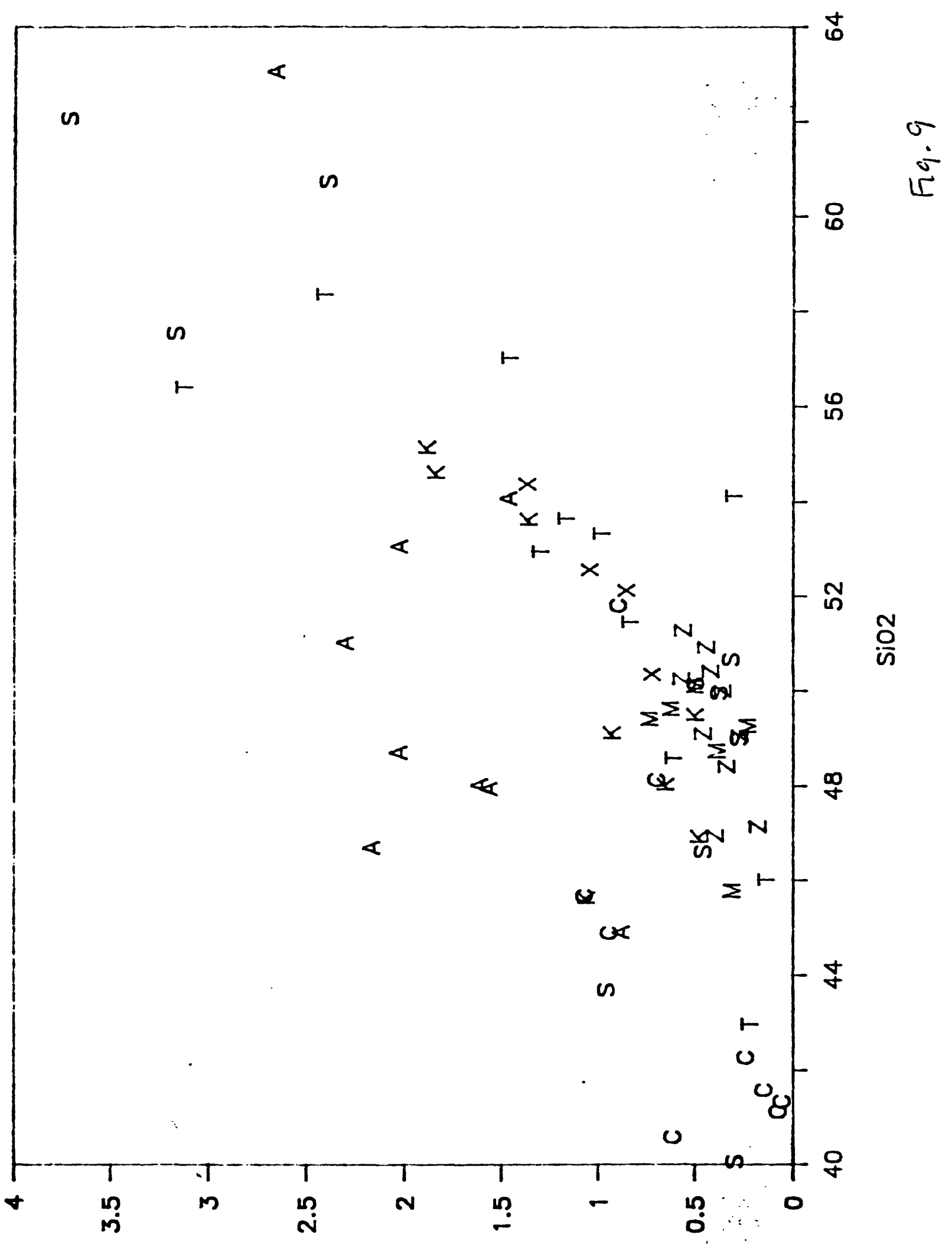

21 


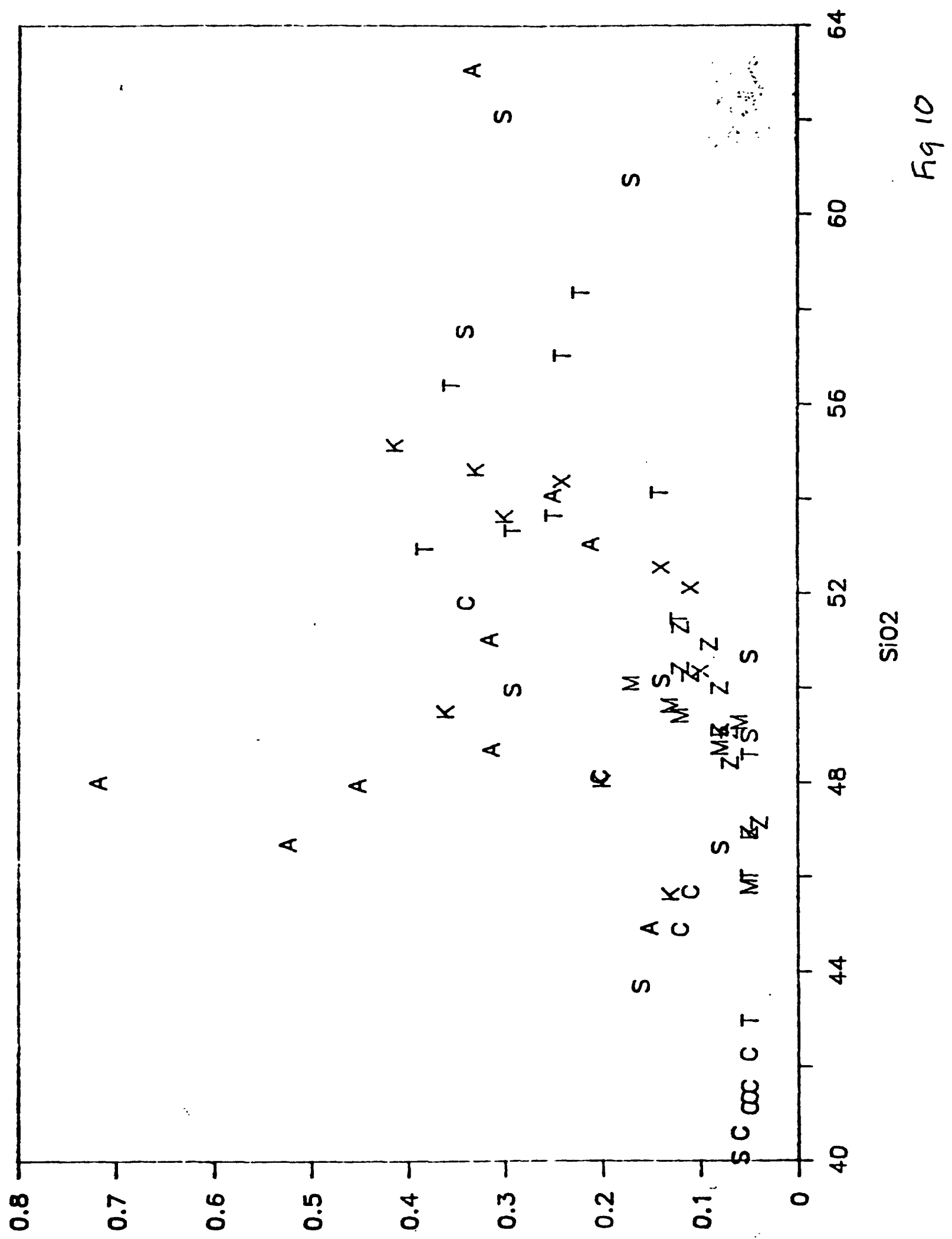

sozd

22 


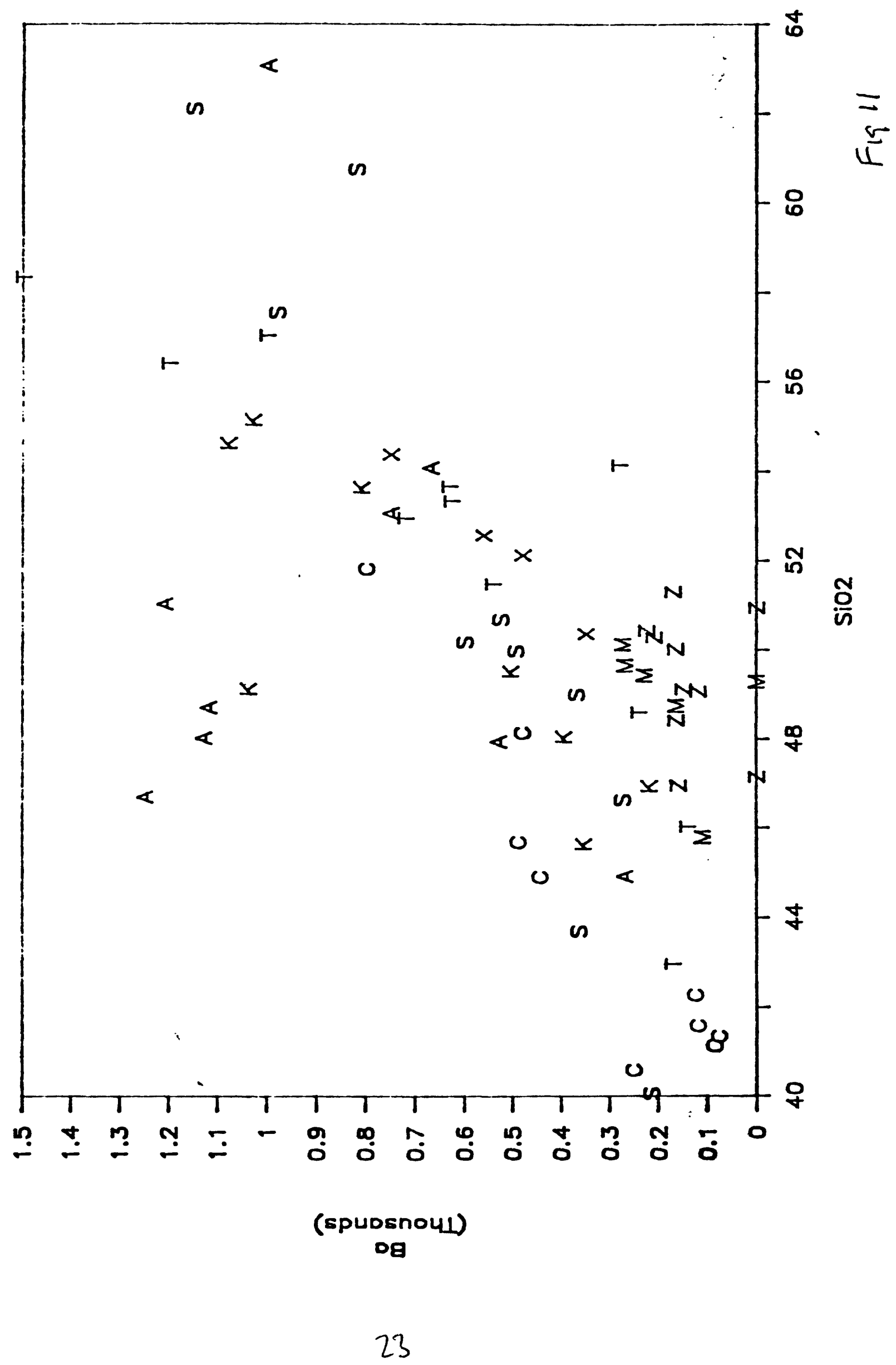




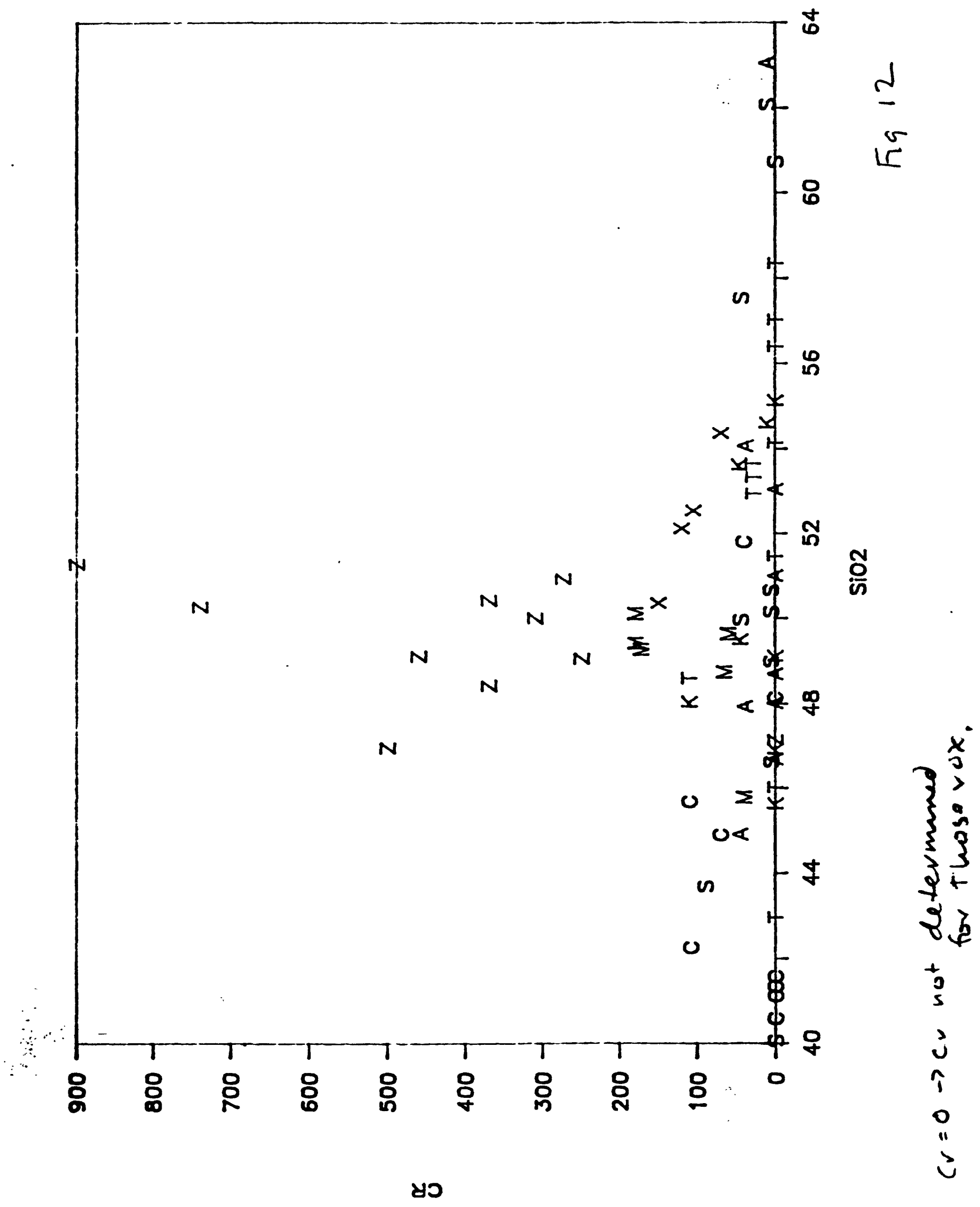




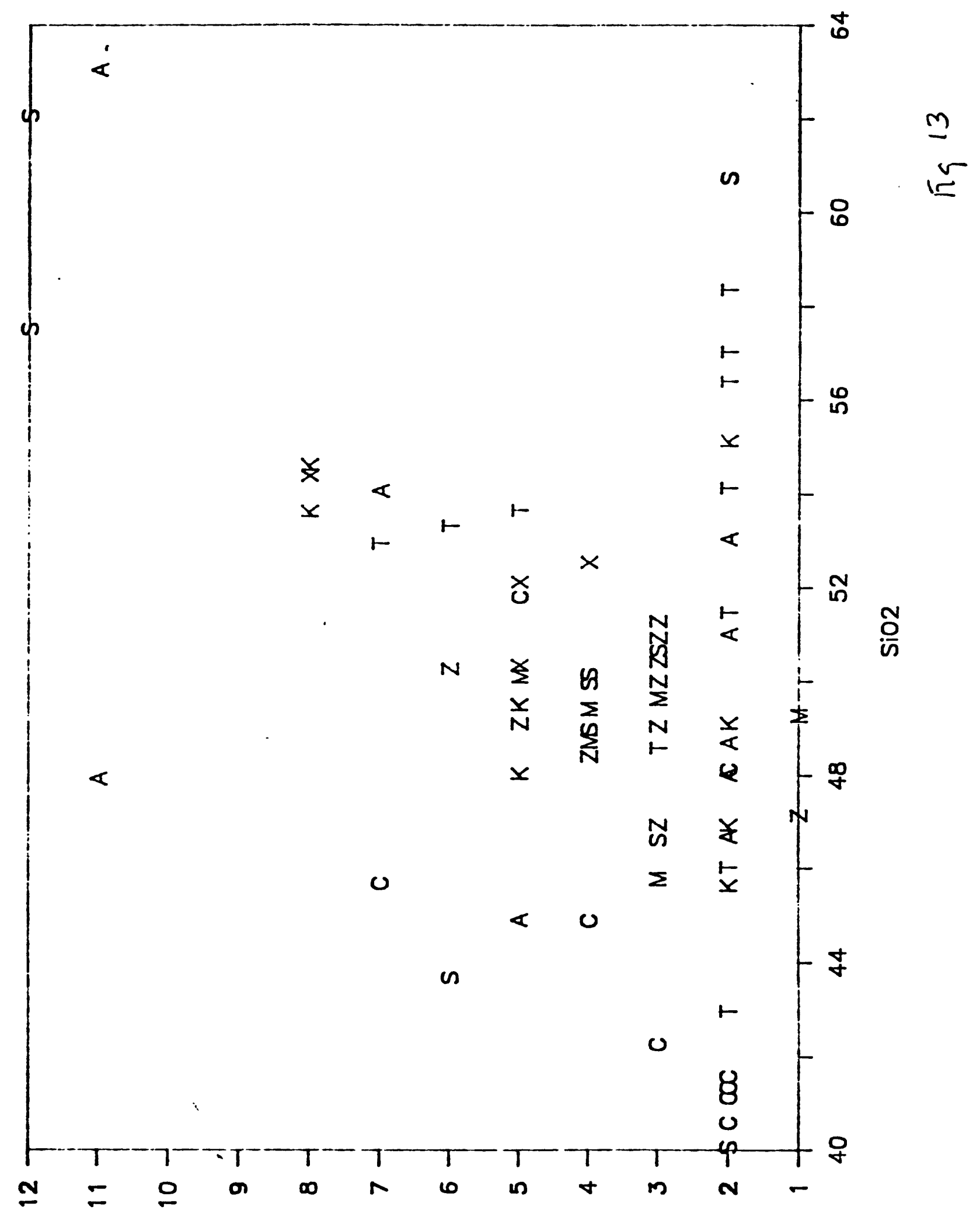

BN

25 


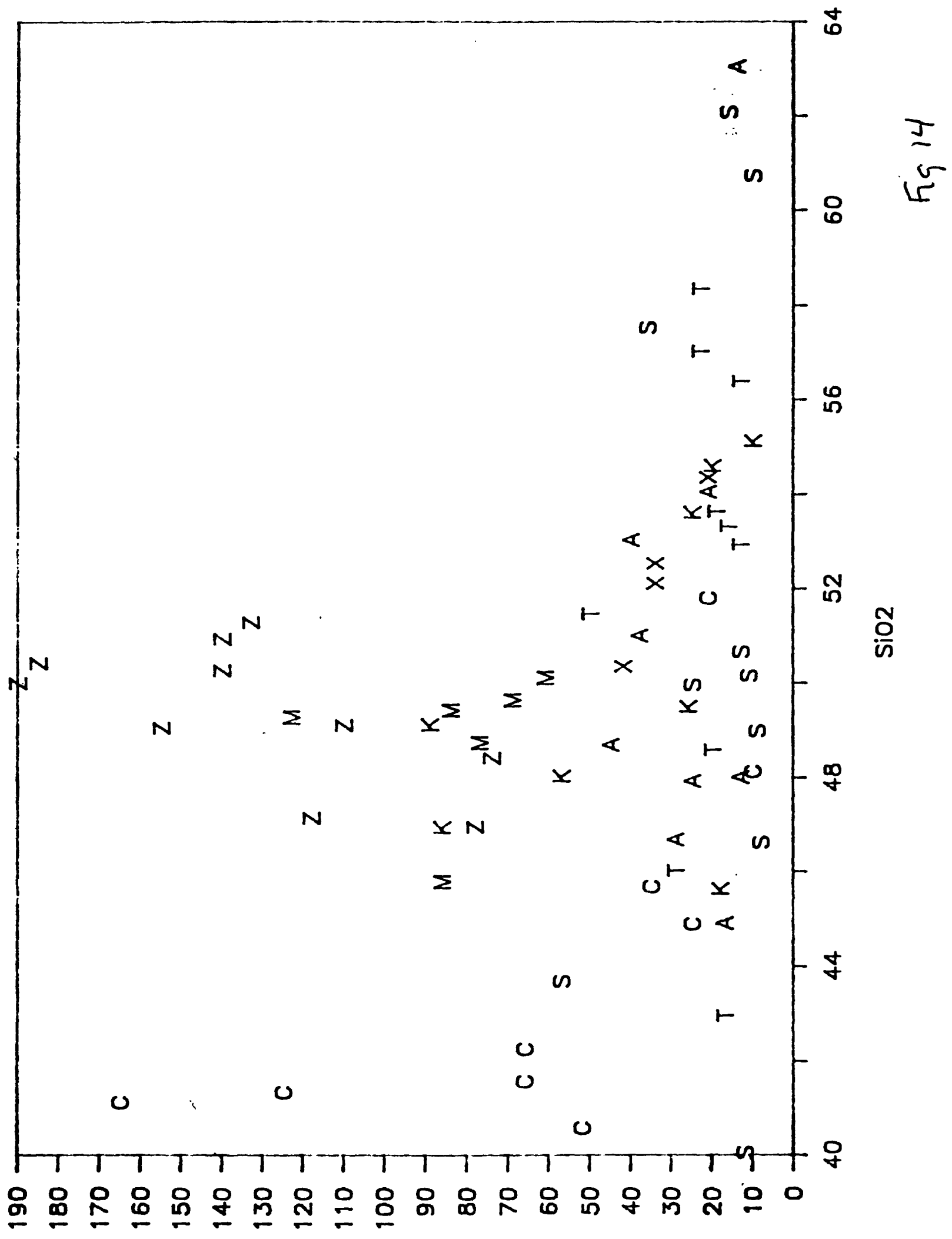

IN

26 


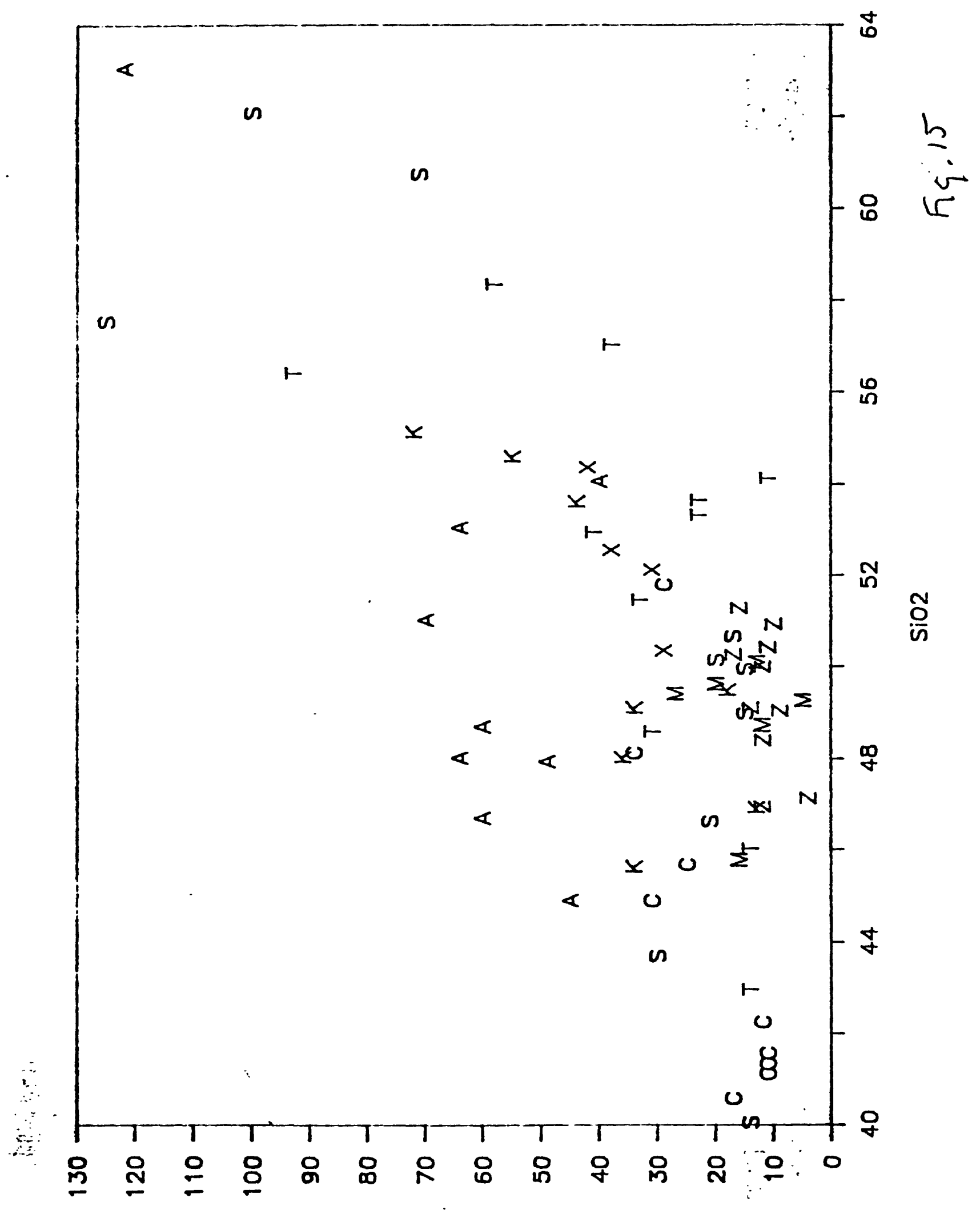

$8 y$ 

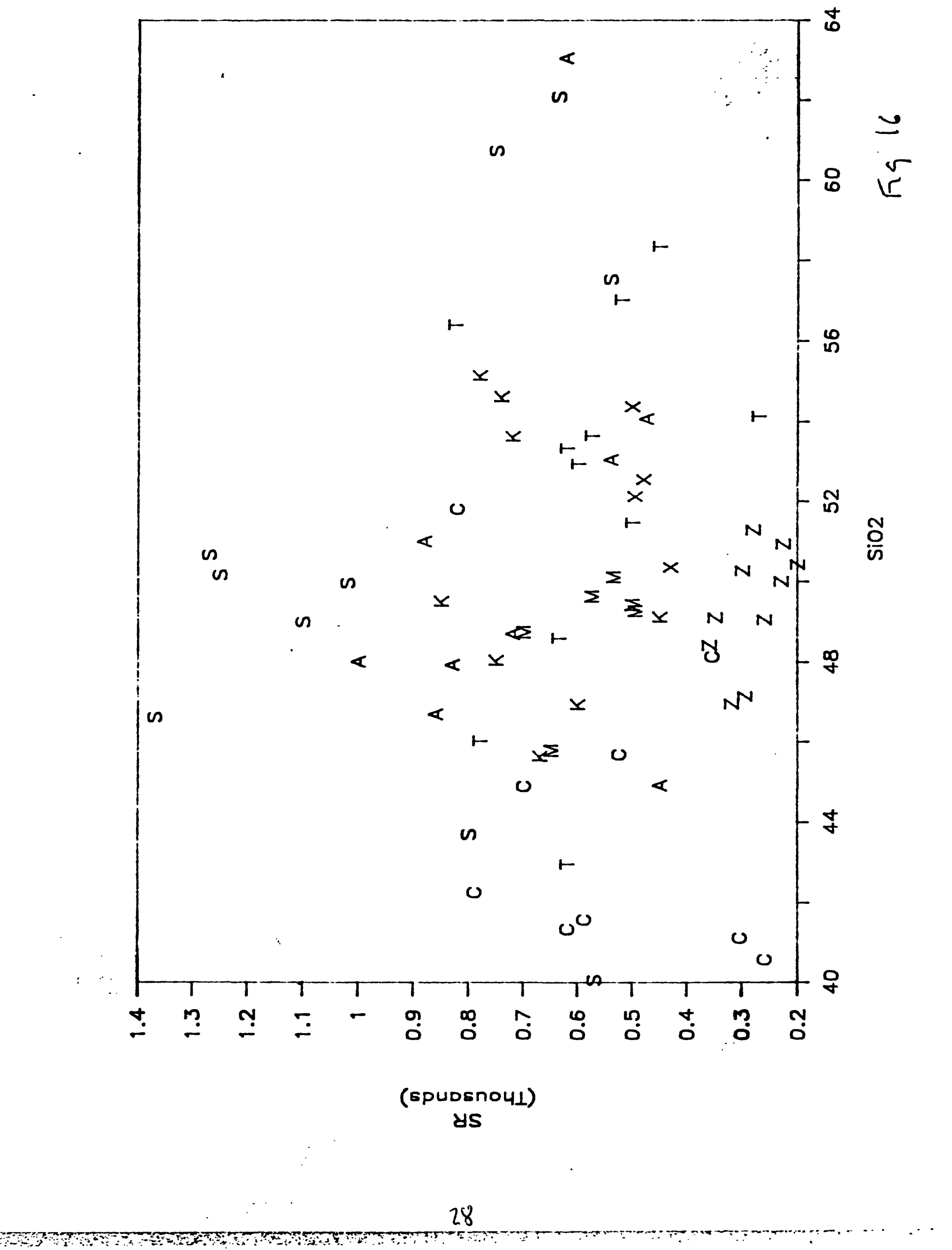


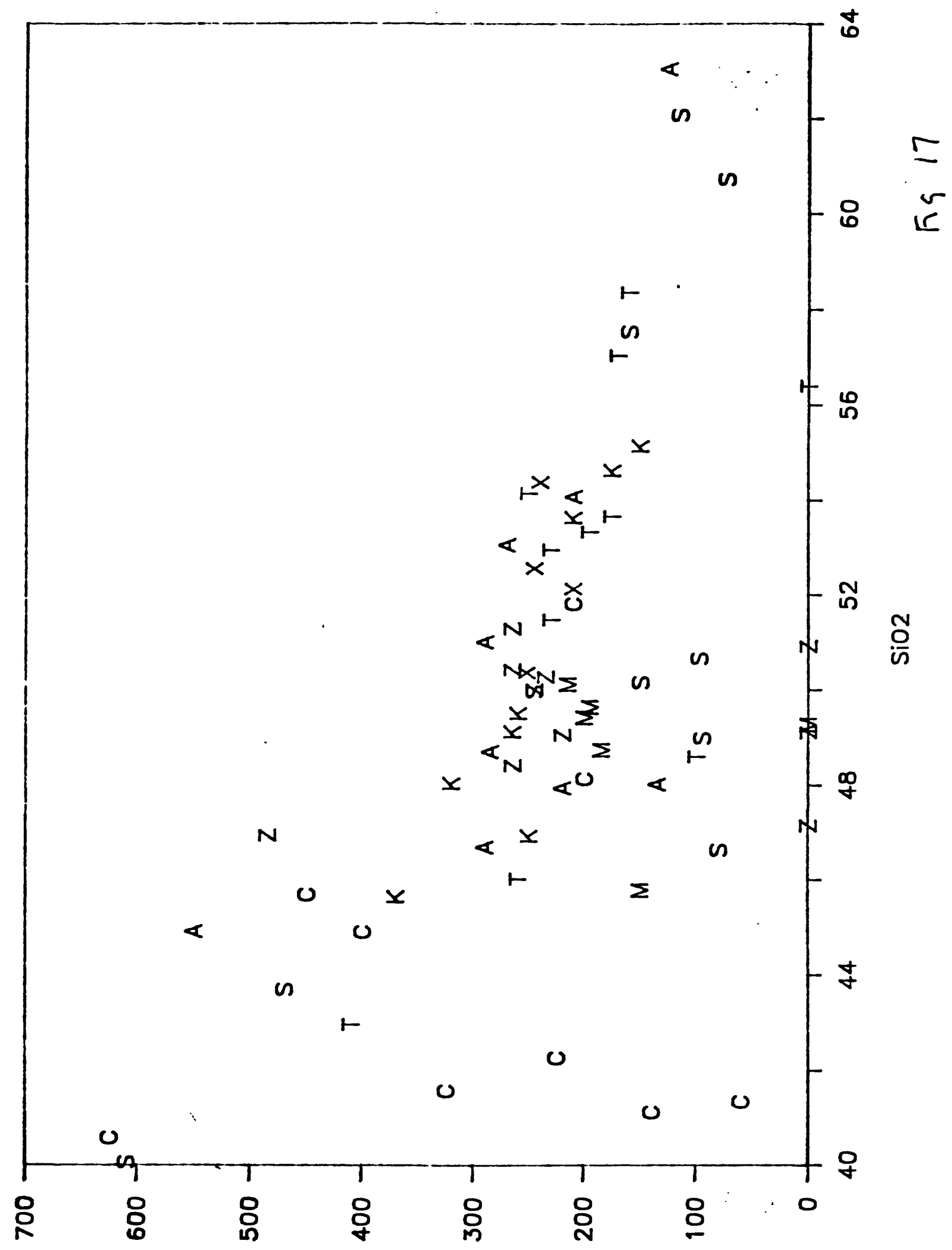

$\wedge$

29 


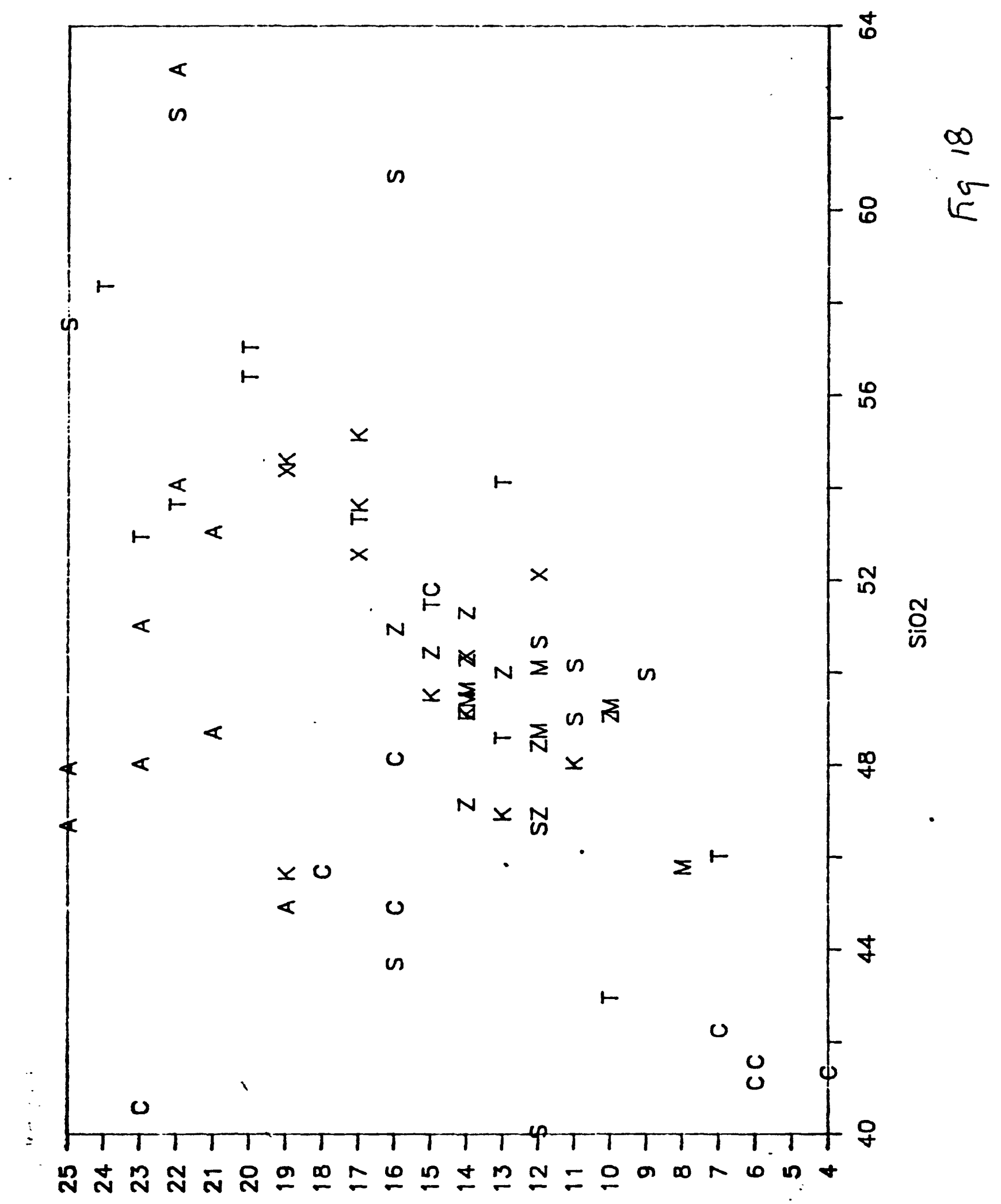

$\lambda$

30 


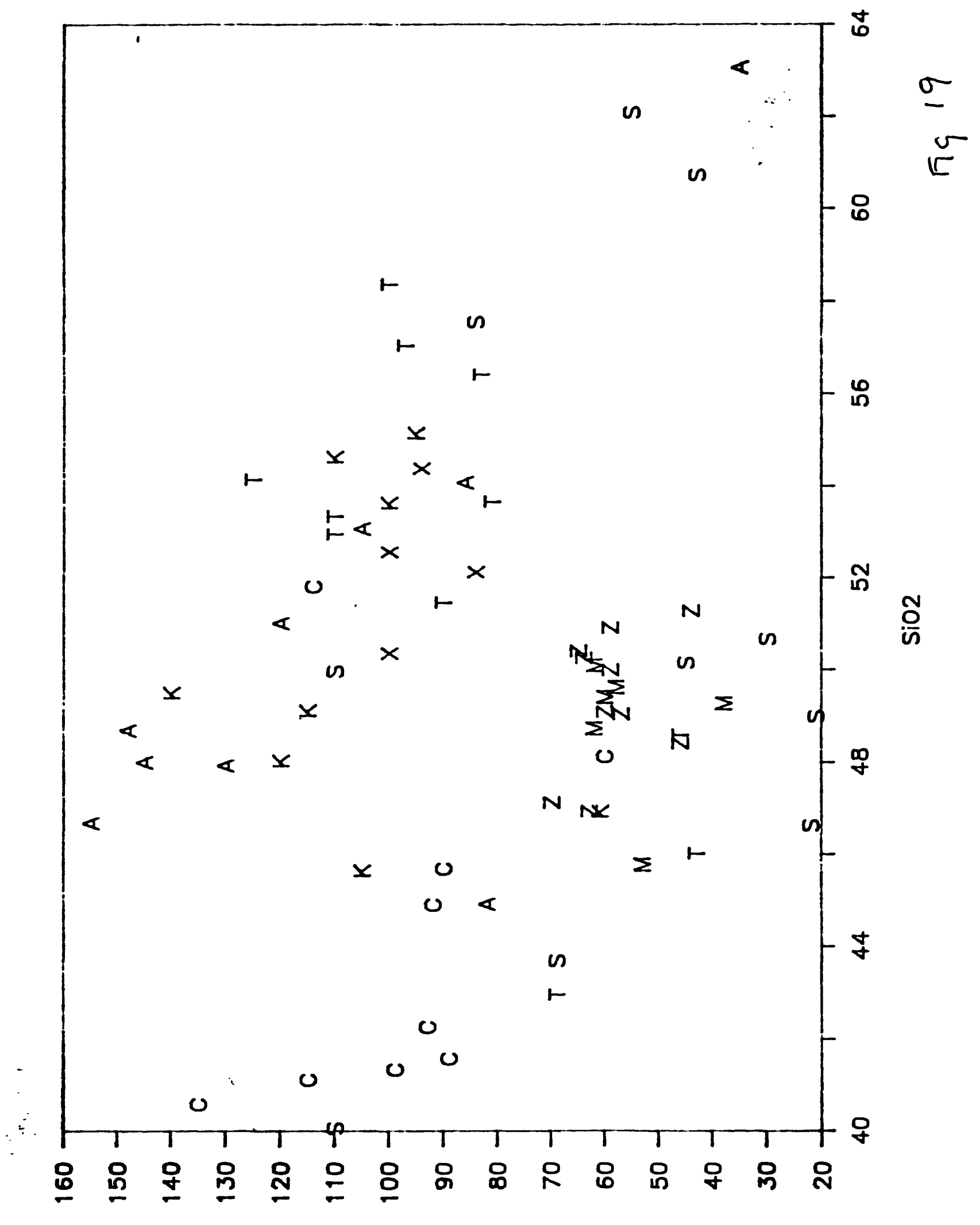

NZ 


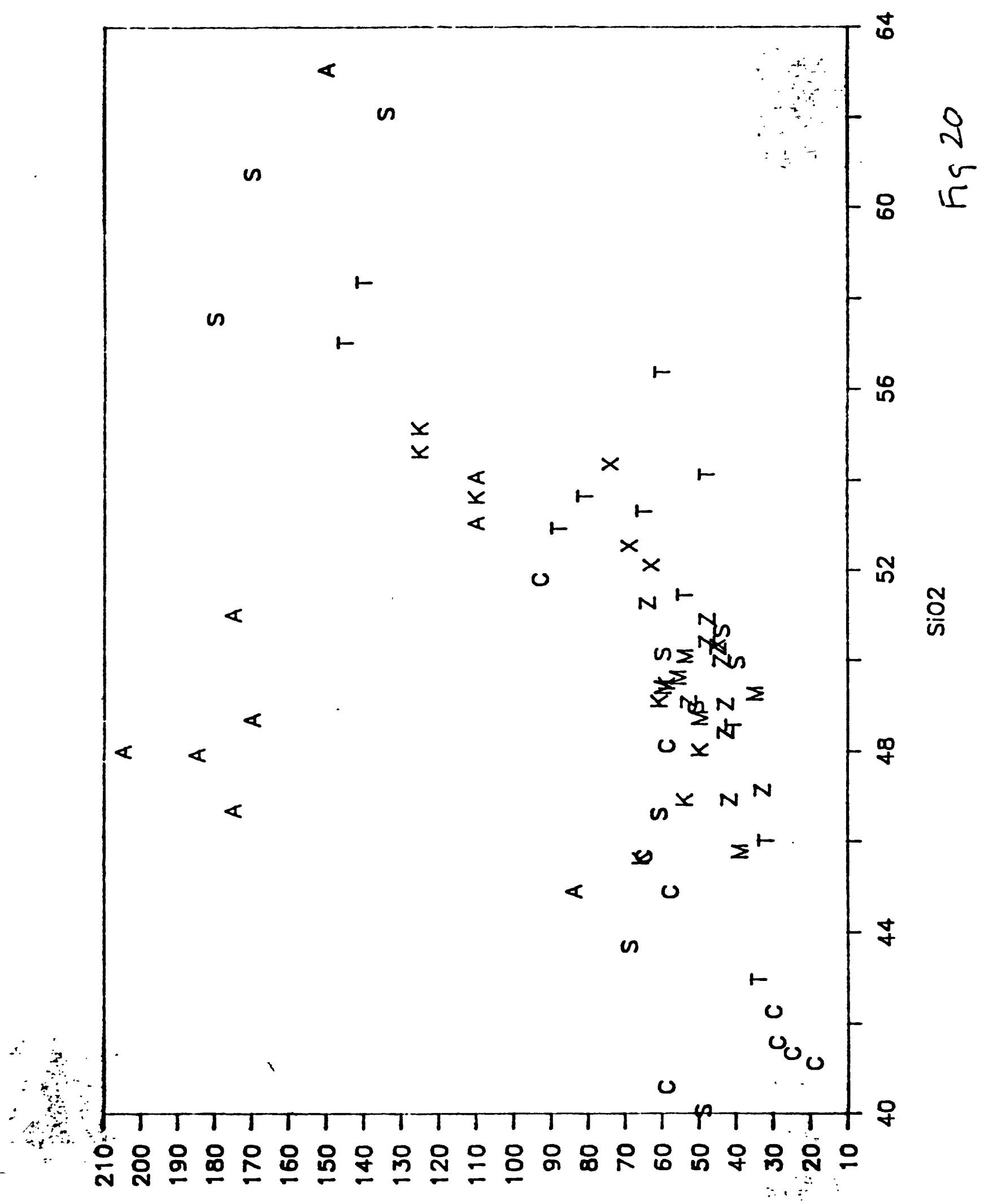

$y z$ 